\title{
Total Variation Spatial Regularization for Sparse Hyperspectral Unmixing
}

\author{
Marian-Daniel Iordache, José M. Bioucas-Dias, Member, IEEE, and Antonio Plaza, Senior Member, IEEE
}

\begin{abstract}
Spectral unmixing aims at estimating the fractional abundances of pure spectral signatures (also called endmembers) in each mixed pixel collected by a remote sensing hyperspectral imaging instrument. In recent work, the linear spectral unmixing problem has been approached in semisupervised fashion as a sparse regression one, under the assumption that the observed image signatures can be expressed as linear combinations of pure spectra, known a priori and available in a library. It happens, however, that sparse unmixing focuses on analyzing the hyperspectral data without incorporating spatial information. In this paper, we include the total variation (TV) regularization to the classical sparse regression formulation, thus exploiting the spatialcontextual information present in the hyperspectral images and developing a new algorithm called sparse unmixing via variable splitting augmented Lagrangian and TV. Our experimental results, conducted with both simulated and real hyperspectral data sets, indicate the potential of including spatial information (through the TV term) on sparse unmixing formulations for improved characterization of mixed pixels in hyperspectral imagery.
\end{abstract}

Index Terms-Hyperspectral imaging, sparse regression, sparse unmixing, spectral unmixing, total variation (TV) regularization.

\section{INTRODUCTION}

$\mathbf{S}$ PECTRAL unmixing is an important technique for hyperspectral data exploitation [1]. Linear spectral unmixing [2] is a standard technique for spectral mixture analysis that infers a set of pure spectral signatures, called endmembers [3], [4], and the fractions of these endmembers, called abundances [5], in each pixel of the scene. This model assumes that the spectra collected by the imaging spectrometer can be expressed in the form of a linear combination of endmembers, weighted by their corresponding abundances. Because each observed spectral signal is the result of an actual mixing process, it is expected that the driving abundances satisfy two constraints, i.e., they should be non-negative [6], and the sum of abundances

Manuscript received July 15, 2011; revised December 26, 2011; accepted February 22, 2012. This work was supported by the European Community's Marie Curie Research Training Networks Programme under contract MRTN-CT-2006-035927, Hyperspectral Imaging Network (HYPERI-NET). Funding from the Spanish Ministry of Science and Innovation (CEOSSPAIN project, reference AYA2011-29334-C02-02) and from the Portuguese Science and Technology Foundation, projects PEst-OE/EEI/LA0008/2011 and PTDC/EEA-TEL/104515/2008, is also gratefully acknowledged.

M.-D. Iordache is with the Flemish Institute for Technological Research (VITO), Centre for Remote Sensing and Earth Observation Processes (TAP), 2400 Mol, Belgium (e-mail: iordache@unex.es).

J. M. Bioucas-Dias is with the Instituto de Telecomunicações, Instituto Superior Técnico, 1049-1 Lisbon, Portugal (e-mail: bioucas@gmail.com).

A. Plaza is with the Hyperspectral Computing Laboratory, Department of Technology of Computers and Communications, Escuela Politécnica, University of Extremadura, 10071 Cáceres, Spain (e-mail: aplaza@unex.es).

Color versions of one or more of the figures in this paper are available online at http://ieeexplore.ieee.org.

Digital Object Identifier 10.1109/TGRS.2012.2191590 for a given pixel should be unity [7]. Although the linear model has practical advantages, such as ease of implementation and flexibility in different applications, nonlinear unmixing describes mixed spectra (in physical [8], [9], or statistical [10] sense) by assuming that part of the source radiation is multiply scattered before being collected at the sensor. The distinction between linear and nonlinear unmixing has been widely studied in recent years [11].

The exploitation of the more general linear mixture model, in spite of its simplicity, has fostered a large amount of research leading to a plethora of endmember identification algorithms based on geometrical and statistical approaches [12]-[37]. A recent trend is the design of algorithms that do not assume the presence of pure signatures in the input data [38]-[42]. However, these algorithms provide virtual endmembers [43] (not necessarily present in the set comprised by input data samples). A recently developed approach to tackle the problems related to the unavailability of pure spectral signatures is to model mixed pixel observations as linear combinations of spectra from a library collected on the ground by a field spectro-radiometer. Unmixing then amounts to finding the optimal subset of signatures in a (potentially very large) spectral library that can best model each mixed pixel in the scene [44]. In practice, this is a combinatorial problem that calls for efficient sparse regression techniques based on sparsity-inducing regularizers, since the number of endmembers participating in a mixed pixel is usually very small compared with the (ever-growing) dimensionality and availability of spectral libraries. To cope with these issues, in previous work we have resorted to fast algorithms such as the sparse unmixing via variable splitting and augmented Lagrangian (SUnSAL) [45] and a constrained version of the same algorithm (CSUnSAL) [45], which exploit the alternating direction method of multipliers (ADMM) [46] in a way similar to [47] and [48].

Sparse unmixing techniques have been increasingly used in recent years. In [49], a new method for subpixel modeling, mapping, and classification of hyperspectral images is presented. It uses learned block-structured discriminative dictionaries [50], where each block is adapted and optimized to represent a specific material in a compact and sparse manner. In [51], a sparsity constraint is included in non-negative matrix factorization, a widely used technique to unmix hyperspectral images and recover the material endmembers [52], [53]. Although sparse unmixing techniques have been shown to exhibit good potential for the characterization of mixed pixels using spectral libraries, they focus mostly on exploiting the spectral information available in the hyperspectral data. Such information deals with pixel vectors in an independent manner from the neighboring pixel values, whereas spatial information concerns the relationship between each pixel vector and its neighbors. 
This means that, despite the inherent spatial-spectral duality that resides in hyperspectral scenes, the classic sparse unmixing approach uses only spectral information and disregards spatialcontextual information. Previous works showed that including spatial information, both as a preprocessing step [54] and in the unmixing procedure itself [55], [56], has a positive impact on the accuracy of the estimated fractional abundances. On the other hand, the success of unmixing when performed via sparse regression techniques relies on the mutual coherence [57] of the spectral library ${ }^{1}$ and the number of materials in the mixtures, i.e., the so-called degree of sparsity [44]. In favorable scenarios, we have low degree of sparsity and low coherence. In hyperspectral applications, the former is often true, but the latter is not.

In order to sidestep the limitations imposed to sparse regression by the high correlation of spectral libraries, in this paper, we develop a new algorithm called sparse unmixing via variable splitting augmented Lagrangian and total variation (SUnSAL-TV). This method includes spatial information on the sparse unmixing formulation by means of the TV regularizer [59]-[61]. This regularizer accounts for spatial homogeneity: it is very likely that two neighboring pixels have similar fractional abundances for the same endmember. The TV regularizer acts as a priori information, which improves the conditioning of the underlying inverse problem [62]. At the end, unmixing is obtained by solving a large nonsmooth convex optimization problem. It should be noted that the work [63] introduces a hyperspectral unmixing approach formally similar to ours. There is, however, a major conceptual difference: whereas we are solving a sparse regression problem based on a library, in [63], the endmembers are jointly inferred with the fractional abundances. Recall that the main rationale for our approach is avoiding the endmember estimation step. A vector TV was also used in [60] to unmix and increase the resolution of hyperspectral images. Again, there is a major conceptual difference with respect to our approach: whereas we are solving a sparse regression problem based on a library, the endmembers in [60] are inferred using an external algorithm, in this case the N-FINDR [17].

The remainder of the paper is structured as follows. Section II describes the linear versus the sparse unmixing formulations. Section III describes the newly developed SUnSAL-TV algorithm. Section IV describes our experimental results with simulated hyperspectral data sets. Section V describes experiments with real hyperspectral data. Section VI concludes with some remarks and hints at plausible future research lines.

\section{Linear Versus Sparse UnMiXing}

\section{A. Linear Spectral Unmixing}

The linear mixture model assumes that the spectral response of a pixel is a linear combination of all the pure spectral signatures (endmembers) present in the pixel. For each pixel, the linear model can be written as follows:

$$
y_{i}=\sum_{j=1}^{q} m_{i j} \alpha_{j}+n_{i}
$$

\footnotetext{
${ }^{1}$ The mutual coherence of a library is closely related with the so-called restricted isometric property [58],
}

where $y_{i}$ is the measured value of the reflectance at spectral band $i, m_{i j}$ is the reflectance of the $j$ th endmember at spectral band $i, \alpha_{j}$ is the fractional abundance of the $j$ th endmember, and $n_{i}$ represents the error term for the spectral band $i$ (i.e., the noise affecting the measurement process). If we assume that the hyperspectral sensor used for data acquisition has $L$ spectral bands, (1) can be rewritten in compact form as:

$$
\mathbf{y}=\mathbf{M} \boldsymbol{\alpha}+\mathbf{n}
$$

where $\mathbf{y}$ is an $L \times 1$ column vector (the measured spectrum of the pixel), $\mathbf{M}$ is an $L \times q$ matrix containing $q$ endmembers, $\alpha$ is a $q \times 1$ vector containing the fractional abundances of the endmembers in the pixel, and $\mathbf{n}$ is an $L \times 1$ vector collecting the errors affecting the measurements at each spectral band. The socalled abundance non-negativity constraint (ANC): $\alpha_{i} \geq 0$ for $i=1, \ldots, q$, and the abundance sum-to-one constraint (ASC): $\sum_{i=1}^{q} \alpha_{i}=1$, respectively, represented in compact form by $\boldsymbol{\alpha} \geq \mathbf{0}$ and $\mathbf{1}^{T} \boldsymbol{\alpha}=1$ (where $\mathbf{1}^{T}$ is a line vector of 1's compatible with $\boldsymbol{\alpha}$ ) are often imposed into the model described in (1), owing to the fact that $\alpha_{i}$, for $i=1, \ldots, q$, represent the fractions of the endmembers present in the considered pixel [5]. In a typical hyperspectral unmixing scenario, we are given a set $\mathbf{Y} \equiv\left\{\mathbf{y}_{i} \in \mathbb{R}^{L}, i=1, \ldots, n\right\}$ of $n$ observed $L$-dimensional spectral vectors, and the objective is to estimate the endmember matrix $\mathrm{M}$ and the fractional abundances $\alpha$ for every pixel in the scene.

\section{B. Sparse Unmixing}

Sparse unmixing reformulates (2) assuming the availability of a library of spectral signatures a priori as follows:

$$
\mathbf{y}=\mathbf{A x}+\mathbf{n}
$$

where $\mathbf{x}$ is the fractional abundance vector compatible with library $\mathbf{A} \in \mathbb{R}^{L \times m}$. Due to the fact that only a few of the signatures contained in $\mathbf{A}$ are likely contributing to the observed spectrum, $\mathrm{x}$ contains many values of zero, which means that it is sparse. An important indicator regarding the difficulty to infer correct solutions for a linear system of equations is the socalled mutual coherence, defined as the largest cosine between any two columns of $\mathbf{A}$. It has been shown that the quality of the solution of a linear system of equations decreases when the mutual coherence increases. As shown in [44], the mutual coherence of hyperspectral libraries tend to be close to one. In [44], we present detailed formulations of sparse unmixing under the form of different convex optimization problems, for both noiseless and noisy environments. In this paper, we consider only noisy observations due to their most realistic nature. With these considerations in mind, the unmixing problem can be formulated as an $l_{2}-l_{0}$ norm optimization problem, in which the observations are affected by noise and the ANC is enforced

$$
\min _{\mathbf{x}} \frac{1}{2}\|\mathbf{A} \mathbf{x}-\mathbf{y}\|_{2}^{2}+\lambda\|\mathbf{x}\|_{0} \quad \text { subject to } \quad \mathbf{x} \geq \mathbf{0}
$$

where $\|\mathbf{x}\|_{0}$ represents the so-called $l_{0}$ norm of the vector $\mathbf{x}$, which simply counts the nonzero components of $\mathbf{x}$, and $\lambda$ is a regularization parameter which weights the two terms of the objective function. This formulation has a simple interpretation: 
we look for the sparsest non-negative solution $\mathrm{x}$ which perfectly explains the data $\mathbf{y}$, given the spectral library $\mathbf{A}$. It should be noted that, in the SUnSAL-TV formulation developed in this paper, we explicitly enforce the ANC constraint but not the ASC constraint. This is because, following the reasoning in [44], the ASC is prone to strong criticisms in the literature, while the ANC alone automatically imposes a generalized ASC (see [44] for more details). The optimization problem in (4) is equivalent to

$$
\min _{\mathbf{x}}\|\mathbf{x}\|_{0} \quad \text { subject to } \quad \mathbf{A x}=\mathbf{y} \quad \mathbf{x} \geq \mathbf{0} .
$$

Both problems (4) and (5) are nonconvex and difficult to solve. However, under certain conditions [57], the $l_{0}$ norm can be replaced by the $l_{1}$ norm. Moreover, since we are interested in solving problems in which the observations are affected by noise, we allow a small reconstruction error for the solution in (5). With these assumptions in mind, the two objective functions, respectively, become

$$
\begin{aligned}
& \min _{\mathbf{x}} \frac{1}{2}\|\mathbf{A} \mathbf{x}-\mathbf{y}\|_{2}^{2}+\lambda\|\mathbf{x}\|_{1} \quad \text { subject to } \quad \mathbf{x} \geq \mathbf{0} \\
& \min _{\mathbf{x}}\|\mathbf{x}\|_{1} \quad \text { subject to } \quad\|\mathbf{A} \mathbf{x}-\mathbf{y}\|_{2} \leq \delta, \quad \mathbf{x} \geq 0
\end{aligned}
$$

where $\delta$ is the tolerated reconstruction error. The optimization problems in (6) and (7) are convex and equivalent. This means that, for any given parameter $\lambda$, a corresponding parameter $\delta$ can be found which leads to the same unmixing solution. In the following section, we describe a new algorithm called SUnSAL-TV which efficiently solves the sparse unmixing problem taking into account the relationship between each pixel vector and its neighbors, including the TV regularizer [59]-[61] on top of the aforementioned sparse unmixing formulation.

\section{Total VARiation for SPARse UnMiXing-The SUNSAL-TV ALGORITHM}

Let $\mathbf{Y} \in \mathbb{R}^{L \times n}$ be the observed data matrix (where each column contains the observed spectrum of a given pixel); let $\mathbf{X} \in \mathbb{R}^{m \times n}$ be the fractional abundances matrix; let $\|\mathbf{X}\|_{F} \equiv$ $\sqrt{\operatorname{trace}\left\{\mathbf{X X}^{T}\right\}}$ be the Frobenius norm of $\mathbf{X}$; let $\|\mathbf{X}\|_{1,1} \equiv$ $\sum_{i=1}^{n}\left\|\mathbf{x}_{i}\right\|_{1}\left(\mathbf{x}_{i}\right.$ denotes the $i$ th column of $\left.\mathbf{X}\right)$; and let $\lambda \geq 0$ and $\lambda_{T V} \geq 0$ be regularization parameters. With these definitions in place, we can now carry out the sparse unmixing by solving the following optimization problem

$$
\begin{aligned}
& \min _{\mathbf{X}} \frac{1}{2}\|\mathbf{A X}-\mathbf{Y}\|_{F}^{2}+\lambda\|\mathbf{X}\|_{1,1}+\lambda_{T V} T V(\mathbf{X}), \\
& \text { subject to } \quad \mathbf{X} \geq 0
\end{aligned}
$$

where

$$
T V(\mathbf{X}) \equiv \sum_{\{i, j\} \in \varepsilon}\left\|\mathbf{x}_{i}-\mathbf{x}_{j}\right\|_{1}
$$

is a vector extension of the nonisotropic TV [59], [61], which promotes piecewise constant (or smooth) transitions in the fractional abundance of the same endmember among neighboring pixels, and $\varepsilon$ denotes the set of horizontal and vertical neighbors in the image. The minimization in (8) with $\lambda_{T V}$ set to zero is a constrained basis pursuit denoising (CBPDN) problem [64] applied to each individual pixel. The application of CBPDN and several other constrained sparse regression algorithms to hyperspectral unmixing was extensively studied in [44].

It should be noted that the optimization problem in (8), although convex, is very hard to solve owing to nonsmooth terms and its huge dimensionality. For instance, if we consider a $256 \times 256$-pixel image and a spectral library with 500 materials, then $\mathbf{X} \in \mathbb{R}^{500 \times 256^{2}}$, which is about 40 million variables! To solve the problem in (8), we have modified the SUnSAL algorithm [45] following closely the methodology introduced in [62]. The core idea is to introduce a set of new variables per regularizer and then use the ADMM method [46] to solve the resulting constrained optimization problem. By a careful choice of the new variables, the initial problem is converted into a sequence of much simpler problems. Next, we formalize the SUnSAL-TV algorithm (more details are given in an Appendix at the end of the paper).

Let $\mathbf{H}_{h}: \mathbb{R}^{m \times n} \rightarrow \mathbb{R}^{m \times n}$ denote a linear operator computing the horizontal differences between the components of $\mathbf{X}$ corresponding to neighboring pixels; i.e., $\mathbf{H}_{h} \mathbf{X}=$ $\left[\mathbf{d}_{1}, \mathbf{d}_{2}, \ldots, \mathbf{d}_{n}\right]$, where $\mathbf{d}_{i}=\mathbf{x}_{i}-\mathbf{x}_{i_{h}}$, with $i$ and $i_{h}$ denoting a pixel and its horizontal neighbor. We are assuming periodic boundaries. Let $\mathbf{H}_{v}: \mathbb{R}^{m \times n} \rightarrow \mathbb{R}^{m \times n}$ be defined in a similar way for the vertical diferences; i.e., $\mathbf{H}_{v} \mathbf{X}=\left[\mathbf{v}_{1}, \mathbf{v}_{2}, \ldots, \mathbf{v}_{n}\right]$, where $\mathbf{v}_{i}=\mathbf{x}_{i}-\mathbf{x}_{i_{v}}$, with $i$ and $i_{v}$ denoting a pixel and its vertical neighbor. With these two difference operators, we define

$$
\mathbf{H X} \equiv\left[\begin{array}{l}
\mathbf{H}_{h} \mathbf{X} \\
\mathbf{H}_{v} \mathbf{X}
\end{array}\right] .
$$

With these definitions in place, an equivalent way of writing the optimization problem (8) is

$$
\min _{\mathbf{X}} \frac{1}{2}\|\mathbf{A X}-\mathbf{Y}\|_{F}^{2}+\lambda\|\mathbf{X}\|_{1,1}+\lambda_{T V}\|\mathbf{H X}\|_{1,1}+\iota_{R+}(\mathbf{X})
$$

where $\iota_{R+}(\mathbf{X})=\sum_{i=1}^{n} \iota_{R+}\left(\mathbf{x}_{i}\right)$ is the indicator function $\left(\mathbf{x}_{i}\right.$ represents the $i$ th column of $\mathbf{X}$ and $\iota_{R+}\left(\mathbf{x}_{i}\right)$ is zero if $\mathbf{x}_{i}$ belongs to the nonnegative orthant and $+\infty$ otherwise).

Given the objective function (10), we write the following (constrained) equivalent formulation:

$$
\begin{aligned}
& \min _{\mathbf{U}, \mathbf{V}_{1}, \mathbf{V}_{2}, \mathbf{V}_{3}, \mathbf{V}_{4}, \mathbf{V}_{5}} \frac{1}{2}\left\|\mathbf{V}_{1}-\mathbf{Y}\right\|_{F}^{2}+\lambda\left\|\mathbf{V}_{2}\right\|_{1,1}+\lambda_{T V}\left\|\mathbf{V}_{4}\right\|_{1,1} \\
&+\iota_{R+}\left(\mathbf{V}_{5}\right), \\
& \text { subject to } \quad \mathbf{V}_{1}=\mathbf{A} \mathbf{U} \\
& \mathbf{V}_{2}=\mathbf{U} \\
& \mathbf{V}_{3}=\mathbf{U} \\
& \mathbf{V}_{4}=\mathbf{H} \mathbf{V}_{3} \\
& \mathbf{V}_{5}=\mathbf{U} .
\end{aligned}
$$

In (11), notice the asymmetry of the constraint $\mathbf{V}_{4}=\mathbf{H V}_{3}$, compared with the remaining constraints. As seen below, this asymmetry underlies large computational gains by decoupling the optimization in the spatial domain from the optimization in the spectral domain. Optimization (11) can be written in a compact form as follows:

$$
\min _{\mathbf{U}, \mathbf{V}} g(\mathbf{V}) \quad \text { subject to } \quad \mathbf{G} \mathbf{U}+\mathbf{B V}=0
$$




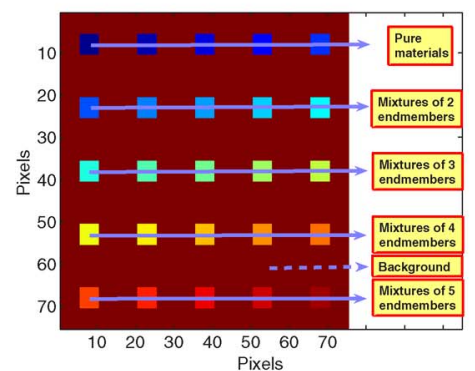

(a)

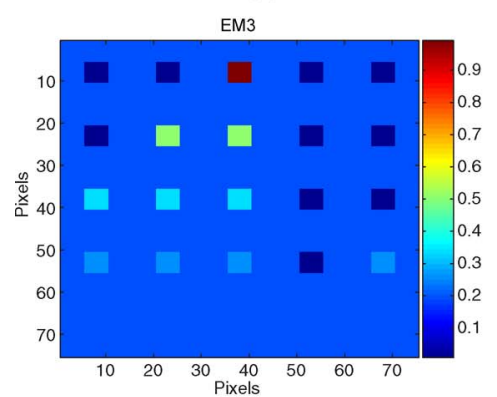

(d)

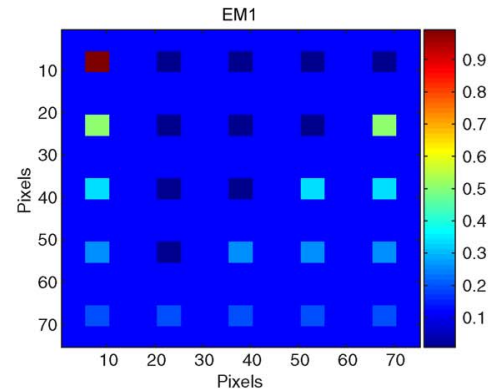

(b)

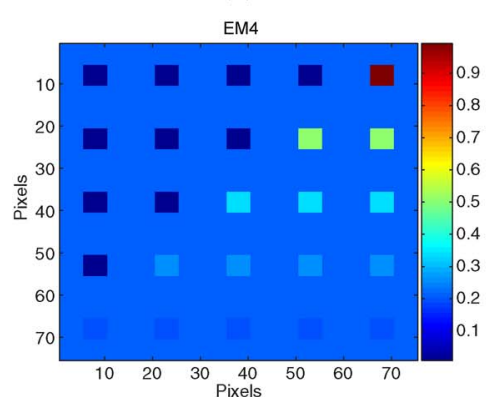

(e)

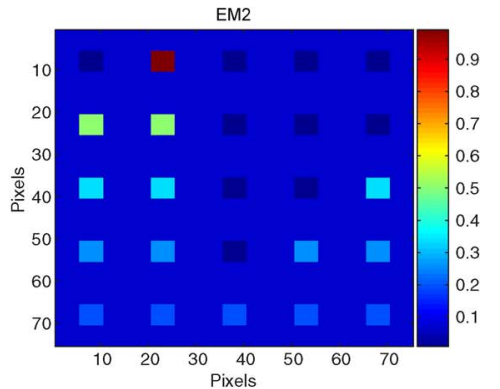

(c)

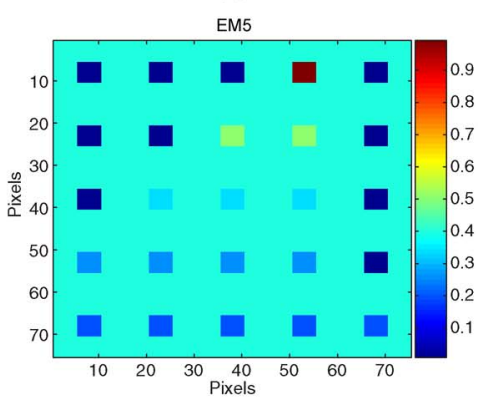

(f)

Fig. 1. True fractional abundances of endmembers in the simulated data cube 1 (DC1). (a) Simulated image; (b) abundances of endmember; (c) abundances of endmember; (d) abundances of endmember; (e) abundances of endmember; (f) abundances of endmember.

where

$$
\begin{aligned}
\mathbf{V} & \equiv\left(\mathbf{V}_{1}, \mathbf{V}_{2}, \mathbf{V}_{3}, \mathbf{V}_{4}, \mathbf{V}_{5}\right), \\
g(\mathbf{V}) & \equiv \frac{1}{2}\left\|\mathbf{V}_{1}-\mathbf{Y}\right\|_{F}^{2}+\lambda\left\|\mathbf{V}_{2}\right\|_{1,1}+\lambda_{T V}\left\|\mathbf{V}_{4}\right\|_{1,1}+\iota_{R+}\left(\mathbf{V}_{5}\right) \\
\mathbf{G} & =\left[\begin{array}{c}
\mathbf{A} \\
\mathbf{I} \\
\mathbf{I} \\
\mathbf{0} \\
\mathbf{I}
\end{array}\right] \quad \mathbf{B}=\left[\begin{array}{ccccc}
-\mathbf{I} & \mathbf{0} & \mathbf{0} & \mathbf{0} & \mathbf{0} \\
\mathbf{0} & -\mathbf{I} & \mathbf{0} & \mathbf{0} & \mathbf{0} \\
\mathbf{0} & \mathbf{0} & -\mathbf{I} & \mathbf{0} & \mathbf{0} \\
\mathbf{0} & \mathbf{0} & \mathbf{H} & -\mathbf{I} & \mathbf{0} \\
\mathbf{0} & \mathbf{0} & \mathbf{0} & \mathbf{0} & -\mathbf{I}
\end{array}\right]
\end{aligned}
$$

The ADMM algorithm for the formulation (12) is shown in Algorithm 1, where (see [62] and [65]):

$$
\mathcal{L}(\mathbf{U}, \mathbf{V}, \mathbf{D}) \equiv g(\mathbf{U}, \mathbf{V})+\frac{\mu}{2}\|\mathbf{G} \mathbf{U}+\mathbf{B V}-\mathbf{D}\|_{F}^{2}
$$

is the augmented Lagrangian for problem (12), $\mu>0$ is a positive constant, and $\mathbf{D} / \mu$ denotes the Lagrange multipliers associated to the constraint $\mathbf{G U}+\mathbf{B V}=0$. In each iteration, Algorithm 1 sequentially optimizes $\mathcal{L}$ with respect to $\mathbf{U}$ (step 3 ) and $\mathbf{V}$ (step 4), and then updates the Lagrange multipliers (step 5).

Algorithm 1 ADMM pseudocode for solving problem (12).

1. Initialization: set $k=0$, choose $\mu>0, \mathbf{U}^{(0)}, \mathbf{V}^{(0)}, \mathbf{D}^{(0)}$

\section{2. repeat:}

3. $\mathbf{U}^{(k+1)} \leftarrow \arg \min _{\mathbf{U}} \mathcal{L}\left(\mathbf{U}, \mathbf{V}^{(k)}, \mathbf{D}^{(k)}\right)$

4. $\mathbf{V}^{(k+1)} \leftarrow \arg \min _{\mathbf{V}} \mathcal{L}\left(\mathbf{U}^{(k+1)}, \mathbf{V}, \mathbf{D}^{(k)}\right)$

5. $\mathbf{D}^{(k+1)} \leftarrow \mathbf{D}^{(k)}-\mathbf{G} \mathbf{U}^{(k+1)}-\mathbf{B} \mathbf{V}^{(k+1)}$

6. until some stopping criterion is satisfied.

Matrix $\mathbf{G}$ is full column rank and function $g$ introduced in (12) is closed, proper, and convex. Then, [46, Theorem 1] ensures that, for any $\mu>0$, if (12) has a solution, say $\mathbf{U}^{*}$, then the sequence $\left\{\mathbf{U}^{(k)}\right\}$, converges to $\mathbf{U}^{*}$. If (12) does not have a solution, then at least one of the sequences $\left\{\mathbf{U}^{(k)}\right\}$ or $\left\{\mathbf{D}^{(k)}\right\}$ diverges. As stopping criterion, we use $\left\|\mathbf{G} \mathbf{U}^{(k)}+\mathbf{B V}^{(k)}\right\|_{F} \leq$ $\varepsilon$. The convergence speed of the ADMM algorithm depends on a suitable choice of parameter $\mu$. This is an active research topic. For example, previous work in [66], [67] (see also [68]) uses an adaptive scheme (based on the primal and the dual ADMM variables) that performs very well in our case. In this scheme, $\mu$ is updated with the objective of keeping the ratio between the ADMM primal and dual residual norms within a given positive interval, as they both converge to zero.

We present in the Appendix the details of the optimizations with respect to $\mathbf{U}$ and $\mathbf{V}$ of the ADMM Algorithm 1, which we term SUnSAL-TV. Here, we make, however, a few pertinent observations. The optimization $\mathbf{U}$ amounts at solving a linear system of equations of size $m \times m$. The matrix involved in this system of equations is fixed and then can be precomputed involving low complexity as the rank of $\mathbf{A}$ is $\min \{L, m\}$. The optimization with respect to $\mathbf{V}$ is decoupled with respect to $\mathbf{V}_{1}$, $\mathbf{V}_{2},\left(\mathbf{V}_{3}, \mathbf{V}_{4}\right)$, and $\mathbf{V}_{5}$. The reason for the coupling between $\mathbf{V}_{3}$ and $\mathbf{V}_{4}$ is the asymmetry already referred to in variable splitting introduced in (11). If we had followed [62] exactly, we would have $\mathbf{V}_{3}=\mathbf{H U}$ instead of the couple $\mathbf{V}_{3}=\mathbf{U}$ and $\mathbf{V}_{4}=\mathbf{H V}_{3}$. However, in this case, the optimization with respect to $\mathbf{U}$ would be unbearable, as we would have matrix $\mathbf{A}$ acting over the spectral dimension and matrix $\mathbf{H}$ acting over the spatial domain in the same system.

As shown in the Appendix, the optimizations with respect to $\mathbf{V}_{3}$ alone and to $\mathbf{V}_{4}$ alone are very light (the same is true for $\mathbf{V}_{1}, \mathbf{V}_{2}$, and $\mathbf{V}_{5}$ ). Therefore, a very simple way to achieve joint optimization with respect to $\left(\mathbf{V}_{3}, \mathbf{V}_{4}\right)$ is to cycle over these two optimizations until convergence. In our SUnSAL-TV algorithm we cycle only once. We have two reasons for this decision: 1) there is no need for an exact solution of optimizations with respect to $\mathbf{U}$ (step 3 ) and $\mathbf{V}$ (step 4) [46, Theorem 1], as long as the errors are summed; and 2) we have observed systematically faster convergence with just one step than with more steps. A 


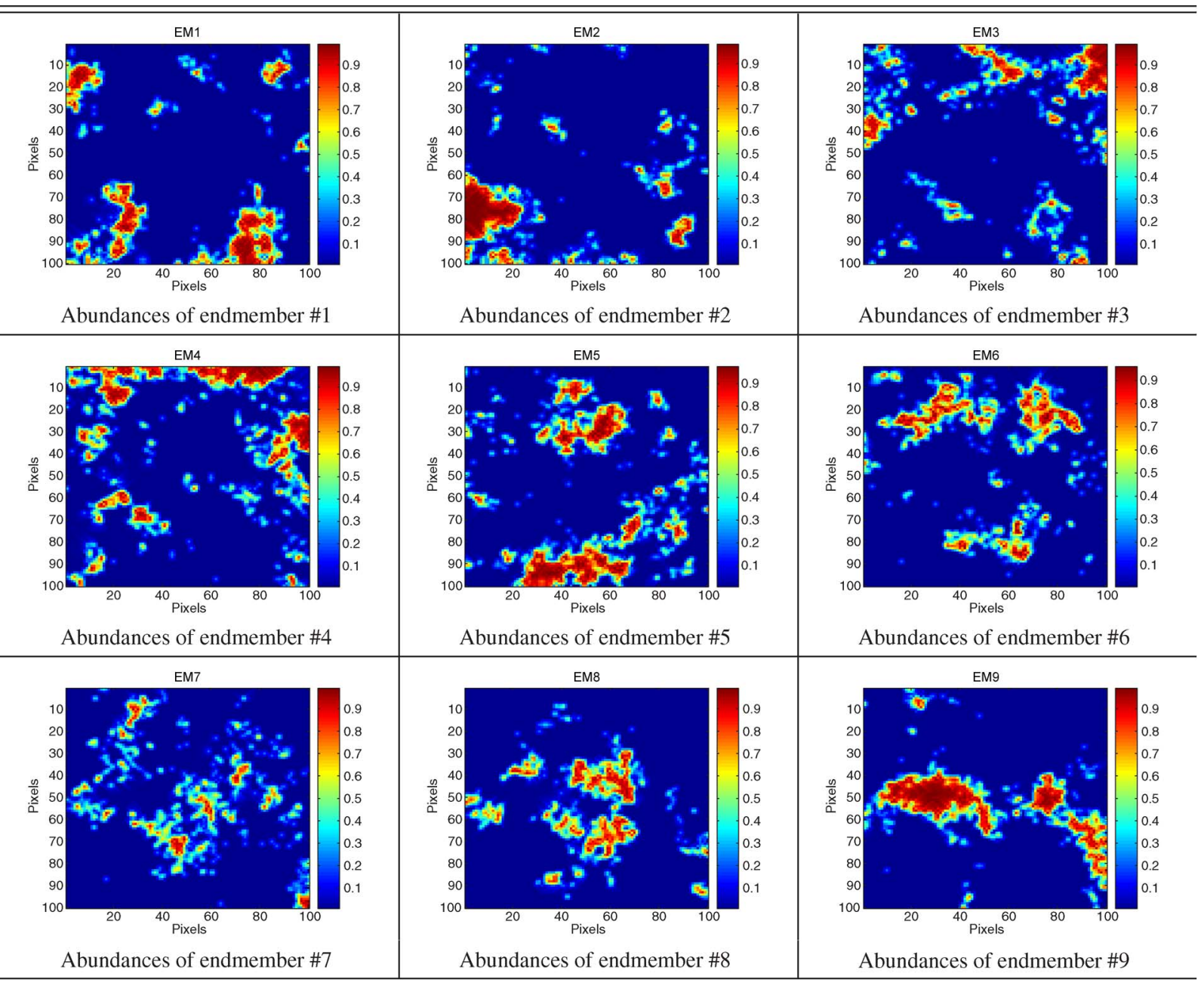

Fig. 2. True fractional abundances of endmembers in the simulated data cube 2 (DC2).

proof of the convergence of SUnSAL-TV with just one step is, however, beyond the scope of this paper.

Concerning computational complexity, we show in the Appendix that each iteration of SUnSAL-TV has complexity $\mathcal{O}\left(L^{2} n\right)+\mathcal{O}(L n \log n)$. Given that $L$ is usually larger than $n \log n$, the first term dominates the total complexity of the algorithm.

Before concluding this section, it is important to emphasize that, in this work, we test the SUnSAL-TV formulation using an exhaustive range of values for parameters $\lambda$ and $\lambda_{T V}$, including zero. Hereinafter, we term the problem in (8) with $\lambda=0$ as nonnegative constrained least squares TV (NCLS-TV) and only nonnegative constrained least squares (NCLS) [5] when $\lambda=\lambda_{T V}=0$.

In the following sections, we provide experimental results using both simulated and real hyperspectral data sets to illustrate the potential advantages of including TV regularization on top of sparse unmixing formulations, including both SUnSAL and NCLS.

\section{EXPERIMENTS With Simulated DATA}

In this section, we illustrate the unmixing performance achieved by including the TV regularizer on top of sparse regression formulations for spectral unmixing using two simulated hyperspectral data sets.
The goal is to analyze the influence of the TV regularizer in the unmixing results, both for solutions which are explicitly constrained to be sparse, and also for the ones computed without enforcing the sparsity explicitly. The section is organized as follows. Section IV-A describes how the simulated data sets have been generated. We consider only scenes affected by noise, as the noiseless case is trivial. Section IV-B describes the considered performance discriminators. Section IV-C concludes with a summary of the most relevant aspects observed in our simulated data experiments.

\section{A. Simulated Data Sets}

We have considered two spectral libraries in our simulated image experiments: $\mathbf{A}_{1} \in \mathbb{R}^{224 \times 240}$ and $\mathbf{A}_{2} \in \mathbb{R}^{224 \times 230}$. The first library is generated from a random selection of 240 materials (different mineral types) from the USGS library, denoted splib06 ${ }^{2}$ and released in September 2007. It comprises spectral signatures with reflectance values given in 224 spectral bands and distributed uniformly in the interval $0.4-2.5 \mu \mathrm{m}$. The second library is obtained from a random selection of 230 materials from the ASTER library, ${ }^{3}$ a compilation of over 2400 spectra

\footnotetext{
${ }^{2}$ Available online: http://speclab.cr.usgs.gov/spectral.lib06

${ }^{3}$ Available online: http://speclib.jpl.nasa.gov
} 
This article has been accepted for inclusion in a future issue of this journal. Content is final as presented, with the exception of pagination.

TABLE I

SRE(dB) Values Achieved After Applying Different Unmixing Methods to Simulated Data (the Optimal Parameters For Which the Reported Values Were ACHIEved Are Indicated in the Parentheses)

\begin{tabular}{|c|c|c|c|c|c|}
\hline \multicolumn{6}{|c|}{ White noise } \\
\hline Data cube & SNR (dB) & $\begin{array}{c}\text { NCLS } \\
\left(\lambda=0, \lambda_{T V}=0\right)\end{array}$ & $\begin{array}{c}\text { NCLS-TV } \\
\left(\lambda=0, \lambda_{T V} \neq 0\right)\end{array}$ & $\begin{array}{c}\text { SUnSAL } \\
\left(\lambda \neq 0, \lambda_{T V}=0\right)\end{array}$ & $\begin{array}{c}\text { SUnSAL-TV } \\
\left(\lambda \neq 0, \lambda_{T V} \neq 0\right)\end{array}$ \\
\hline \multirow{3}{*}{$\mathrm{DC} 1$} & 20 & 2.1376 & $\begin{array}{c}10.6206 \\
\left(\lambda_{T V}=0.05\right)\end{array}$ & $\begin{array}{c}3.8954 \\
(\lambda=0.05)\end{array}$ & $\begin{array}{c}10.6280 \\
\left(\lambda=5 \cdot 10^{-4} ; \lambda_{T V}=0.05\right)\end{array}$ \\
\hline & 30 & 7.5037 & $\begin{array}{c}11.7573 \\
\left(\lambda_{T V}=0.01\right)\end{array}$ & $\begin{array}{c}8.9476 \\
(\lambda=0.01)\end{array}$ & $\begin{array}{c}11.7677 \\
\left(\lambda=5 \cdot 10^{-4} ; \lambda_{T V}=0.01\right)\end{array}$ \\
\hline & 40 & 10.048 & $\begin{array}{c}12.6539 \\
\left(\lambda_{T V}=0.01\right)\end{array}$ & $\begin{array}{c}10.944 \\
(\lambda=0.005)\end{array}$ & $\begin{array}{c}12.6753 \\
\left(\lambda=5 \cdot 10^{-4} ; \lambda_{T V}=0.005\right)\end{array}$ \\
\hline \multirow{3}{*}{ DC2 } & 20 & 1.5103 & $\begin{array}{c}7.4233 \\
\left(\lambda_{T V}=0.001\right)\end{array}$ & $\begin{array}{c}8.395 \\
(\lambda=0.01)\end{array}$ & $\begin{array}{c}8.6504 \\
\left(\lambda=5 \cdot 10^{-4} ; \lambda_{T V}=0.005\right)\end{array}$ \\
\hline & 30 & 7.9458 & $\begin{array}{c}10.1713 \\
\left(\lambda_{T V}=5 \cdot 10^{-4}\right)\end{array}$ & $\begin{array}{c}10.0148 \\
(\lambda=0.005)\end{array}$ & $\begin{array}{c}11.1713 \\
\left(\lambda=0.001 ; \lambda_{T V}=5 \cdot 10^{-4}\right)\end{array}$ \\
\hline & 40 & 10.6254 & $\begin{array}{c}13.0528 \\
\left(\lambda_{T V}=5 \cdot 10^{-4}\right)\end{array}$ & $\begin{array}{c}12.561 \\
\left(\lambda=5 \cdot 10^{-4}\right)\end{array}$ & $\begin{array}{c}13.0528 \\
\left(\lambda=0 ; \lambda_{T V}=5 \cdot 10^{-4}\right)\end{array}$ \\
\hline \multicolumn{6}{|c|}{ Correlated noise } \\
\hline Data cube & SNR $(\mathrm{dB})$ & $\begin{array}{c}\text { NCLS } \\
\left(\lambda=0, \lambda_{T V}=0\right)\end{array}$ & $\begin{array}{c}\text { NCLS-TV } \\
\left(\lambda=0, \lambda_{T V} \neq 0\right)\end{array}$ & $\begin{array}{c}\text { SUnSAL } \\
\left(\lambda \neq 0, \lambda_{T V}=0\right)\end{array}$ & $\begin{array}{c}\text { SUnSAL-TV } \\
\left(\lambda \neq 0, \lambda_{T V} \neq 0\right)\end{array}$ \\
\hline \multirow{3}{*}{$\mathrm{DC} 1$} & 20 & -1.1976 & $\begin{array}{c}7.4708 \\
\left(\lambda_{T V}=0.05\right)\end{array}$ & $\begin{array}{c}1.8754 \\
(\lambda=0.05)\end{array}$ & $\begin{array}{c}8.6050 \\
\left(\lambda=5 \cdot 10^{-4} ; \lambda_{T V}=0.05\right)\end{array}$ \\
\hline & 30 & 4.9894 & $\begin{array}{c}8.9662 \\
\left(\lambda_{T V}=0.05\right)\end{array}$ & $\begin{array}{c}8.3862 \\
(\lambda=0.01)\end{array}$ & $\begin{array}{c}10.1839 \\
\left(\lambda=5 \cdot 10^{-4} ; \lambda_{T V}=0.01\right)\end{array}$ \\
\hline & 40 & 8.0207 & $\begin{array}{c}11.4320 \\
\left(\lambda_{T V}=0.01\right)\end{array}$ & $\begin{array}{c}11.4620 \\
(\lambda=0.005)\end{array}$ & $\begin{array}{c}11.6856 \\
\left(\lambda=5 \cdot 10^{-4} ; \lambda_{T V}=5 \cdot 10^{-4}\right)\end{array}$ \\
\hline \multirow{3}{*}{$\mathrm{DC} 2$} & 20 & 1.0655 & $\begin{array}{c}4.2458 \\
\left(\lambda_{T V}=0.01\right)\end{array}$ & $\begin{array}{c}2.8945 \\
(\lambda=0.005)\end{array}$ & $\begin{array}{c}5.912 \\
\left(\lambda=0.001 ; \lambda_{T V}=0.001\right)\end{array}$ \\
\hline & 30 & 5.9806 & $\begin{array}{c}10.654 \\
\left(\lambda_{T V}=5 \cdot 10^{-4}\right)\end{array}$ & $\begin{array}{c}7.8206 \\
(\lambda=0.001)\end{array}$ & $\begin{array}{c}11.186 \\
\left(\lambda=5 \cdot 10^{-4} ; \lambda_{T V}=5 \cdot 10^{-4}\right)\end{array}$ \\
\hline & 40 & 12.4347 & $\begin{array}{c}14.6485 \\
\left(\lambda_{T V}=5 \cdot 10^{-4}\right)\end{array}$ & $\begin{array}{c}12.561 \\
\left(\lambda=5 \cdot 10^{-4}\right)\end{array}$ & $\begin{array}{c}14.6485 \\
\left(\lambda=0 ; \lambda_{T V}=5 \cdot 10^{-4}\right)\end{array}$ \\
\hline
\end{tabular}

of natural and man-made materials. Specifically, each of the members of this library has reflectance values measured for 224 spectral bands distributed uniformly in the interval 3-12 $\mu \mathrm{m}$. The mutual coherences [57] of the two libraries are very close to 1. These libraries were used to generate two different simulated hyperspectral data cubes.

1) Simulated Data Cube 1 (DC1): This simulated data cube was generated using five randomly selected spectral signatures from $\mathbf{A}_{1}$. DC1 has $75 \times 75$ pixels and 224 bands per pixel. The data were generated using a linear mixture model, with five randomly selected signatures as the endmembers and imposing the ASC in each simulated pixel. In the resulting simulated image, shown in Fig. 1(a), there are pure regions as well as mixed regions constructed using mixtures ranging between two and five endmembers, distributed spatially in the form of distinct square regions. Fig. 1(b)-(f), respectively, show the true fractional abundances for each of the five endmembers. The background pixels are made up of mixtures of the same five endmembers, but this time their respective fractional abundances values were randomly fixed to values $0.1149,0.0741$, $0.2003,0.2055$, and 0.4051 , respectively. After generating DC1 following the procedure described above, the scene was contaminated with white noise (on the one hand) and also with spectrally correlated noise (on the other hand) resulting from 
This article has been accepted for inclusion in a future issue of this journal. Content is final as presented, with the exception of pagination.

TABLE II

$p_{s}$ Values Values Achieved After Applying Different Unmixing Methods to Simulated Data (the Optimal Parameters For Which the Reported Values Were ACHIEved Are Indicated in the Parentheses)

\begin{tabular}{|c|c|c|c|c|c|}
\hline \multicolumn{6}{|c|}{ White noise } \\
\hline Data cube & SNR (dB) & $\begin{array}{c}\text { NCLS } \\
\left(\lambda=0, \lambda_{T V}=0\right)\end{array}$ & $\begin{array}{c}\text { NCLS-TV } \\
\left(\lambda=0, \lambda_{T V} \neq 0\right)\end{array}$ & $\begin{array}{c}\text { SUnSAL } \\
\left(\lambda \neq 0, \lambda_{T V}=0\right)\end{array}$ & $\begin{array}{c}\text { SUnSAL-TV } \\
\left(\lambda \neq 0, \lambda_{T V} \neq 0\right)\end{array}$ \\
\hline \multirow{3}{*}{ DC1 } & 20 & 0.1076 & $\begin{array}{c}0.9233 \\
\left(\lambda_{T V}=0.05\right)\end{array}$ & $\begin{array}{c}0.4263 \\
(\lambda=0.05)\end{array}$ & $\begin{array}{c}0.9733 \\
\left(\lambda=5 \cdot 10^{-4} ; \lambda_{T V}=0.05\right)\end{array}$ \\
\hline & 30 & 0.8999 & $\begin{array}{c}0.9955 \\
\left(\lambda_{T V}=0.01\right)\end{array}$ & $\begin{array}{c}0.9468 \\
(\lambda=0.01)\end{array}$ & $\begin{array}{c}0.9956 \\
\left(\lambda=5 \cdot 10^{-4} ; \lambda_{T V}=0.01\right)\end{array}$ \\
\hline & 40 & 0.9696 & $\begin{array}{c}0.9955 \\
\left(\lambda_{T V}=0.01\right)\end{array}$ & $\begin{array}{c}0.9877 \\
(\lambda=0.005)\end{array}$ & $\begin{array}{c}0.9956 \\
\left(\lambda=5 \cdot 10^{-4} ; \lambda_{T V}=0.005\right)\end{array}$ \\
\hline \multirow{3}{*}{ DC2 } & 20 & 0.3297 & $\begin{array}{c}0.7986 \\
\left(\lambda_{T V}=0.001\right)\end{array}$ & $\begin{array}{c}0.7446 \\
(\lambda=0.01)\end{array}$ & $\begin{array}{c}0.8395 \\
\left(\lambda=5 \cdot 10^{-4} ; \lambda_{T V}=0.005\right)\end{array}$ \\
\hline & 30 & 0.8044 & $\begin{array}{c}0.8936 \\
\left(\lambda_{T V}=5 \cdot 10^{-4}\right)\end{array}$ & $\begin{array}{c}0.8836 \\
(\lambda=0.005)\end{array}$ & $\begin{array}{c}0.8942 \\
\left(\lambda=0.001 ; \lambda_{T V}=5 \cdot 10^{-4}\right)\end{array}$ \\
\hline & 40 & 0.9133 & $\begin{array}{c}0.9942 \\
\left(\lambda_{T V}=5 \cdot 10^{-4}\right)\end{array}$ & $\begin{array}{c}0.9800 \\
\left(\lambda=5 \cdot 10^{-4}\right)\end{array}$ & $\begin{array}{c}0.9942 \\
\left(\lambda=0 ; \lambda_{T V}=5 \cdot 10^{-4}\right)\end{array}$ \\
\hline \multicolumn{6}{|c|}{ Correlated noise } \\
\hline Data cube & SNR (dB) & $\begin{array}{c}\text { NCLS } \\
\left(\lambda=0, \lambda_{T V}=0\right)\end{array}$ & $\begin{array}{c}\text { NCLS-TV } \\
\left(\lambda=0, \lambda_{T V} \neq 0\right)\end{array}$ & $\begin{array}{c}\text { SUnSAL } \\
\left(\lambda \neq 0, \lambda_{T V}=0\right)\end{array}$ & $\begin{array}{c}\text { SUnSAL-TV } \\
\left(\lambda \neq 0, \lambda_{T V} \neq 0\right)\end{array}$ \\
\hline \multirow{3}{*}{ DC1 } & 20 & 0.0774 & $\begin{array}{c}0.9282 \\
\left(\lambda_{T V}=0.05\right)\end{array}$ & $\begin{array}{c}0.9243 \\
(\lambda=0.05)\end{array}$ & $\begin{array}{c}0.9470 \\
\left(\lambda=5 \cdot 10^{-4} ; \lambda_{T V}=0.05\right)\end{array}$ \\
\hline & 30 & 0.5794 & $\begin{array}{c}0.9726 \\
\left(\lambda_{T V}=0.05\right)\end{array}$ & $\begin{array}{c}0.9244 \\
(\lambda=0.01)\end{array}$ & $\begin{array}{c}0.9732 \\
\left(\lambda=5 \cdot 10^{-4} ; \lambda_{T V}=0.01\right)\end{array}$ \\
\hline & 40 & 0.8967 & $\begin{array}{c}1 \\
\left(\lambda_{T V}=0.01\right)\end{array}$ & $\begin{array}{c}0.9948 \\
(\lambda=0.005)\end{array}$ & $\begin{array}{c}1 \\
\left(\lambda=5 \cdot 10^{-4} ; \lambda_{T V}=5 \cdot 10^{-4}\right)\end{array}$ \\
\hline \multirow{3}{*}{ DC2 } & 20 & 0.3536 & $\begin{array}{c}0.6146 \\
\left(\lambda_{T V}=0.01\right)\end{array}$ & $\begin{array}{c}0.6205 \\
(\lambda=0.005)\end{array}$ & $\begin{array}{c}0.7234 \\
\left(\lambda=0.001 ; \lambda_{T V}=0.001\right)\end{array}$ \\
\hline & 30 & 0.4751 & $\begin{array}{c}0.8942 \\
\left(\lambda_{T V}=5 \cdot 10^{-4}\right)\end{array}$ & $\begin{array}{c}0.8741 \\
(\lambda=0.001)\end{array}$ & $\begin{array}{c}0.9095 \\
\left(\lambda=5 \cdot 10^{-4} ; \lambda_{T V}=5 \cdot 10^{-4}\right)\end{array}$ \\
\hline & 40 & 0.9060 & $\begin{array}{c}0.9818 \\
\left(\lambda_{T V}=5 \cdot 10^{-4}\right)\end{array}$ & $\begin{array}{c}0.9800 \\
\left(\lambda=5 \cdot 10^{-4}\right)\end{array}$ & $\begin{array}{c}0.9918 \\
\left(\lambda=0 ; \lambda_{T V}=5 \cdot 10^{-4}\right)\end{array}$ \\
\hline
\end{tabular}

low-pass filtering i.i.d. Gaussian noise, using a normalized cutoff frequency of $5 \pi / L$, for three levels of the signal-tonoise ratio $\left(\mathrm{SNR} \equiv \mathbb{E}\|\mathbf{A x}\|^{2} / \mathbb{E}\|\mathbf{n}\|_{2}^{2}\right.$ ), i.e., $20 \mathrm{~dB}, 30 \mathrm{~dB}$, and $40 \mathrm{~dB}$.

2) Simulated Data Cube 2(DC2): This simulated data cube contains $100 \times 100$ pixels generated using nine randomly selected signatures from $\mathbf{A}_{2}$. The fractional abundances satisfy the ANC and the ASC and are piecewise smooth, i.e., they are smooth with sharp transitions, as shown in Fig. 2. The resulting observations exhibit spatial homogeneity as described in Fig. 2, which shows the true abundances of the endmembers. After generating DC2 following the procedure described above, the scene was again contaminated with both white and correlated noise using the same SNR values adopted for DC1.

\section{B. Performance Discriminators}

The performance discriminator adopted in this work to measure the quality of the reconstruction of spectral mixtures is the signal to reconstruction error [44]: SRE $\equiv \mathbb{E}\left[\|\mathbf{x}\|_{2}^{2}\right] /$ $\mathbb{E}\left[\|\mathbf{x}-\widehat{\mathbf{x}}\|_{2}^{2}\right]$, measured in dB: SRE $(\mathrm{dB}) \equiv 10 \log _{10}(\mathrm{SRE})$. We use this measure instead of the classical root mean square error 


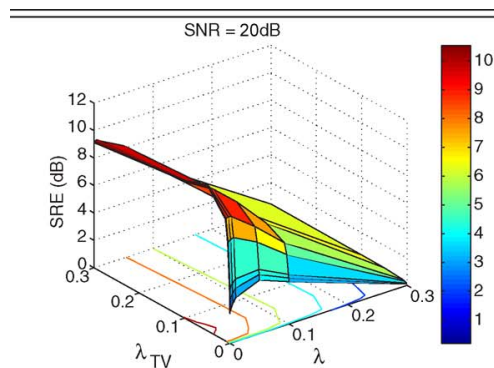

(a)

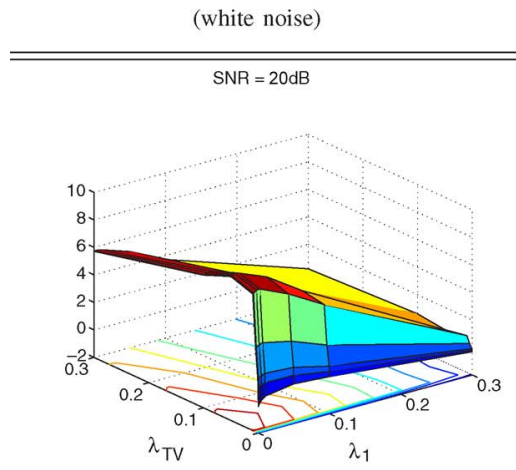

(d)

(correlated noise)

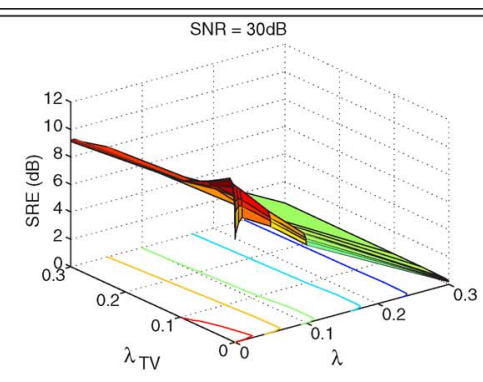

(b)

(white noise)

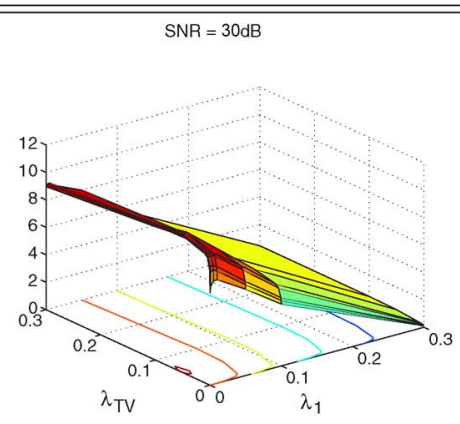

(e)

(correlated noise)

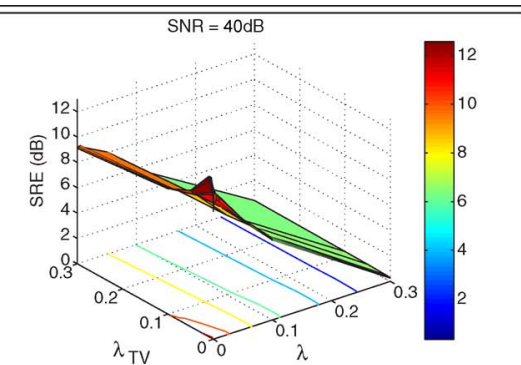

(c)

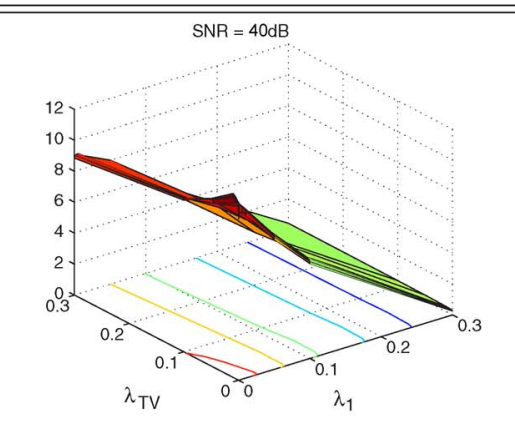

(f)

(correlated noise)

Fig. 3. $\operatorname{SRE}(\mathrm{dB})$ as a function of parameters $\lambda$ and $\lambda_{T V}$ for DC1 with different SNR levels (top: white noise; bottom: correlated noise). (a) SNR $=20 \mathrm{~dB}$ (white noise); (b) SNR $=30 \mathrm{~dB}$ (white noise); (c) SNR $=40 \mathrm{~dB}$ (white noise); (d) SNR $=20 \mathrm{~dB}$ (correlated noise); (e) SNR $=30 \mathrm{~dB}$ (correlated noise); (f) $\mathrm{SNR}=40 \mathrm{~dB}$ (correlated noise).

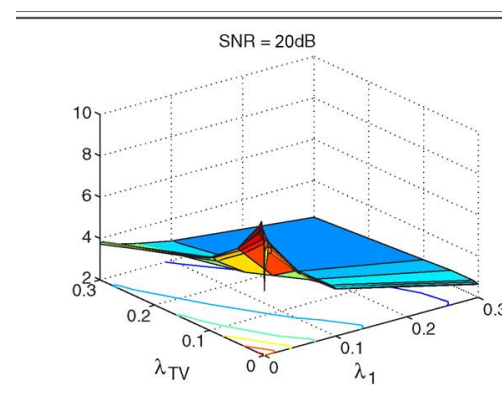

(a)

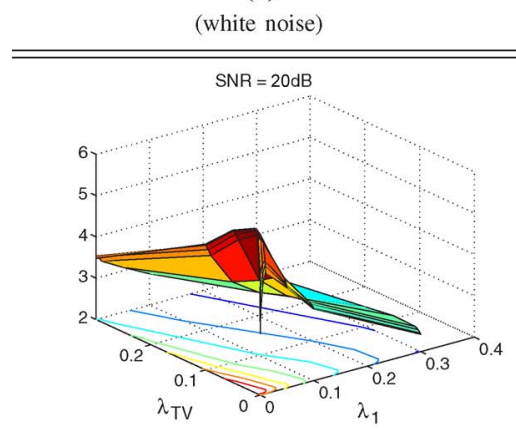

(d)

(correlated noise)

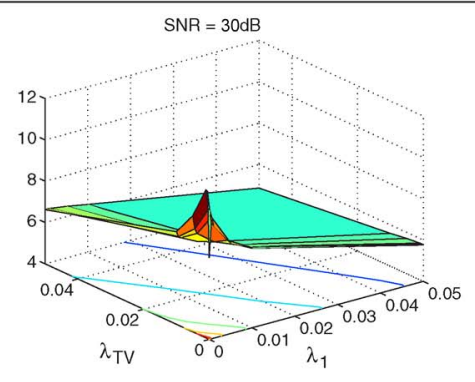

(b)

(white noise)

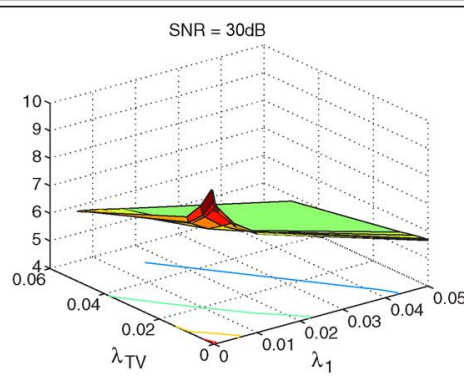

(e)

(correlated noise)

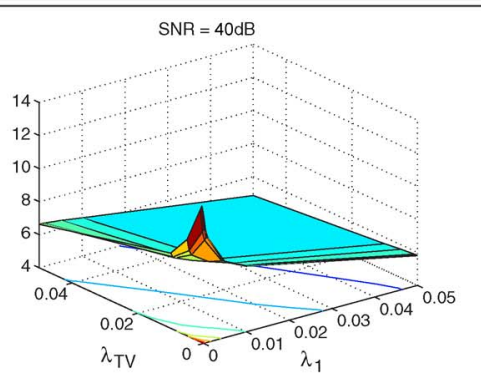

(c)

(white noise)

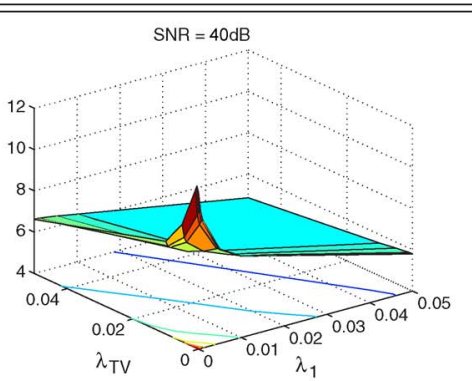

(f)

(correlated noise)

Fig. 4. $\operatorname{SRE}(\mathrm{dB})$ as a function of parameters $\lambda$ and $\lambda_{T V}$ for DC2 with different SNR levels (top: white noise; bottom: correlated noise). (a) SNR $=20 \mathrm{~dB}$ (white noise); (b) SNR = $30 \mathrm{~dB}$ (white noise); (c) SNR = $40 \mathrm{~dB}$ (white noise); (d) SNR $=20 \mathrm{~dB}$ (correlated noise); (e) SNR $=30 \mathrm{~dB}$ (correlated noise); (f) $\mathrm{SNR}=40 \mathrm{~dB}$ (correlated noise).

[54] as it gives more information regarding the power of the error in relation with the power of the signal. The higher the $\mathrm{SRE}(\mathrm{dB})$, the better the unmixing performance.
We also compute a so-called probability of success, $p_{s}$, which is an estimate of the probability that the relative error power be smaller than a certain threshold. This metric is a widespread 


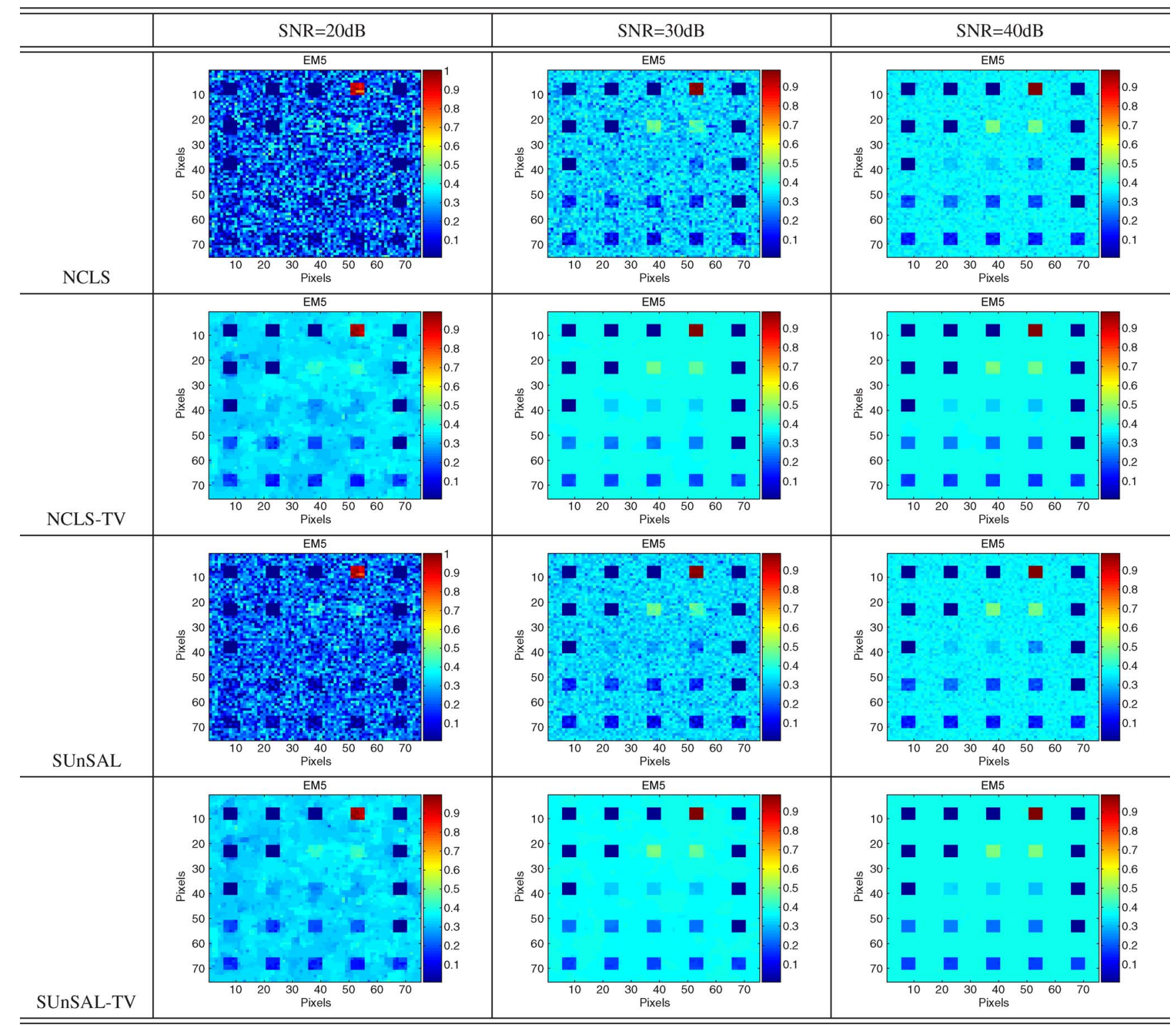

Fig. 5. Abundance maps obtained by different unmixing methods for endmember \#5 in DC1 (white noise).

one in the sparse regression literature, and is formally defined as $p_{s} \equiv P\left(\|\widehat{x}-x\|^{2} /\|x\|^{2} \leq\right.$ threshold $)$. For example, if we set threshold $=10$ and get $p_{s}=1$, this means that the total relative error power of the fractional abundances is, with probability one, less than $1 / 10$. This gives an indication about the stability of the estimation that cannot be directly derived from the SRE (which is an average). In the following, we assume, based on experiments reported in our previous work [44], that the estimation result can be considered successful when $\|\widehat{x}-x\|^{2} /\|x\|^{2} \leq 3.16(5 \mathrm{~dB})$. In all the tests using the TV regularizer on top of sparse unmixing approaches (SUnSALTV and NCLS-TV), we considered a first-order pixel neighborhood system.

\section{Results and Discussion}

In this section, we test the performance of the proposed TV regularizer combined with sparse unmixing formulations using the two simulated data cubes DC1 and DC2. We also include the original SUnSAL and NCLS formulations. The algorithms were tested using different values of the parameters $\lambda$ and $\lambda_{T V}: 0,5 \cdot 10^{-4}, 10^{-3}, 5 \cdot 10^{-3}, 0.01,0.05,0.1,0.3$, 0.5 , and 1 . All possible combinations of these parameters were considered. Table I shows the $\mathrm{SRE}(\mathrm{dB})$ results achieved by the different tested methods with the two considered simulated data sets, using all considered SNR levels. On the other hand, Table II shows the $p_{s}$ results achieved by the different methods for the same data cubes. In both tables, we only report the best scores obtained across the considered parameter range (the optimal parameters for which the reported values were obtained are indicated in the parentheses).

From Tables I and II, we can conclude that the inclusion of the TV regularizer offers the potential to improve unmixing performance in two different analysis scenarios, i.e., when the sparsity is imposed explicitly (SUnSAL), and also when it is not enforced (NCLS). For high SNR values, the improvements 


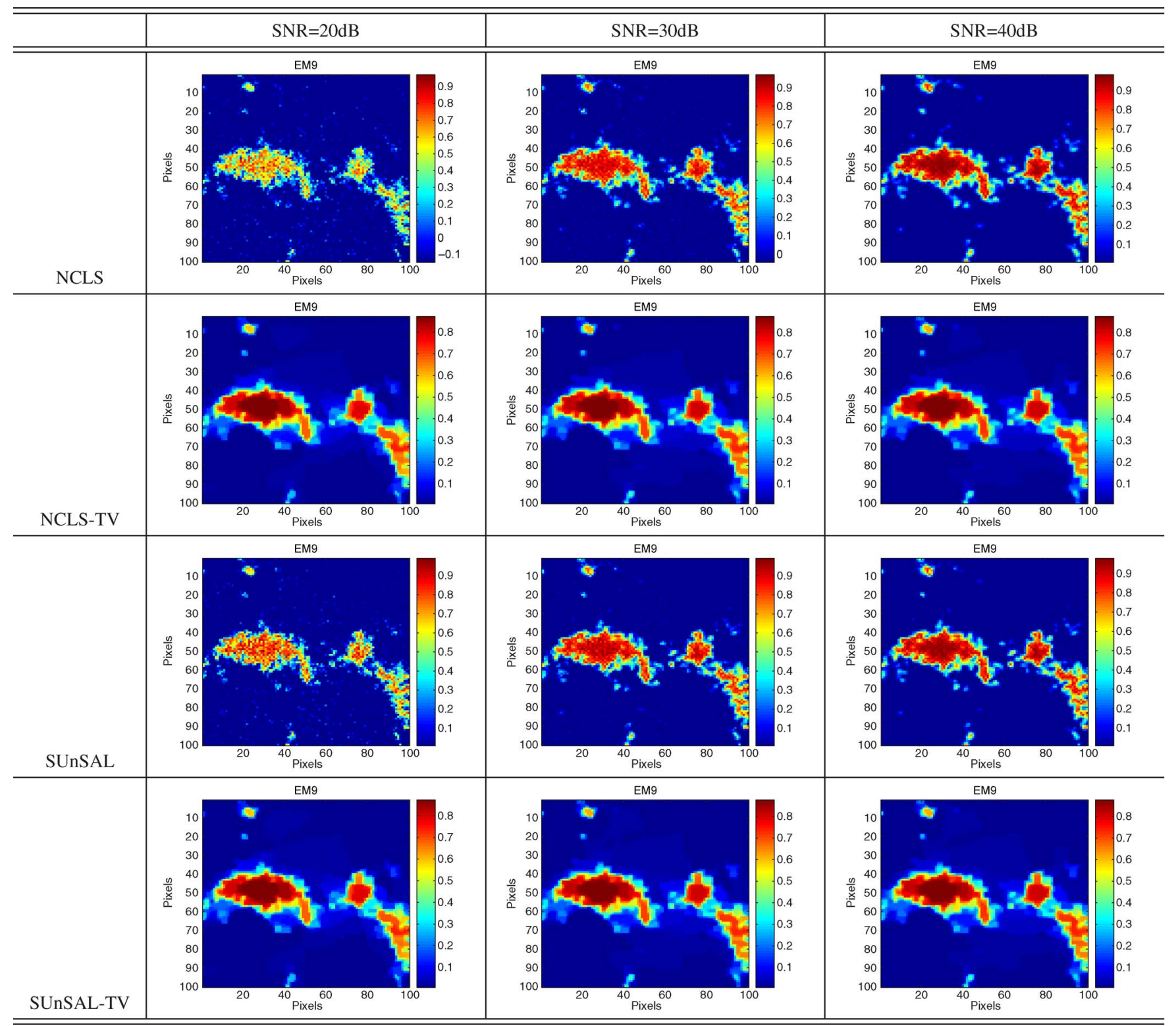

Fig. 6. Abundance maps obtained by different unmixing methods for endmember \#9 in DC2 (white noise).

obtained with regard to the standard sparse unmixing formulations (SUnSAL and NCLS) are not significant. This is due to the fact that, with low noise conditions, NCLS and the $l_{2}-l_{1}$ norm optimization solution are able to recover the fractional abundances with good accuracy. However, as the noise increases, the spatial term becomes more important and improves significantly the quality of unmixing results as it can be observed in the results obtained by SUnSAL-TV and NCLS-TV for SNR $=20 \mathrm{~dB}$ in Tables I and II. Note also that the NCLS-TV and SUnSAL-TV provide results which are not very different, which means that the TV regularizer imposes intrinsically a kind of sparsity in the solutions. Although this is expected to happen, the sparse regularizer is still important as it can be seen in the scenarios dominated by high noise, in which SUnSAL-TV performs clearly better than NCLS-TV.

For illustrative purposes, Figs. 3 (DC1) and 4 (DC2) show the computed values of $\operatorname{SRE}(\mathrm{dB})$ (as a function of both pa- rameters $\lambda$ and $\lambda_{T V}$ ) for observations affected by both white and correlated noise. Here, we do not consider values larger than 0.3 for the two aforementioned parameters as we have experimentally observed that such values lead to poor performances in our experiments. Since the plots obtained for the $p_{s}$ metric are very similar, we do not report them here for space considerations. From Figs. 3 and 4, it can be observed that, for the two considered scenes with different SNR levels, the best unmixing performances were achieved for relatively small values of the parameters. The improvements in unmixing performance resulting from the inclusion of the TV regularizer are more apparent when the SNR is low, while the performance of all methods becomes more similar as the SNR is increased. These observations are in line with those already reported in Tables I and II.

Figs. 5 and 6, respectively, show the abundance maps estimated for one randomly selected endmember in DC1 and DC2 (considering different noise levels). Since the abundance maps 

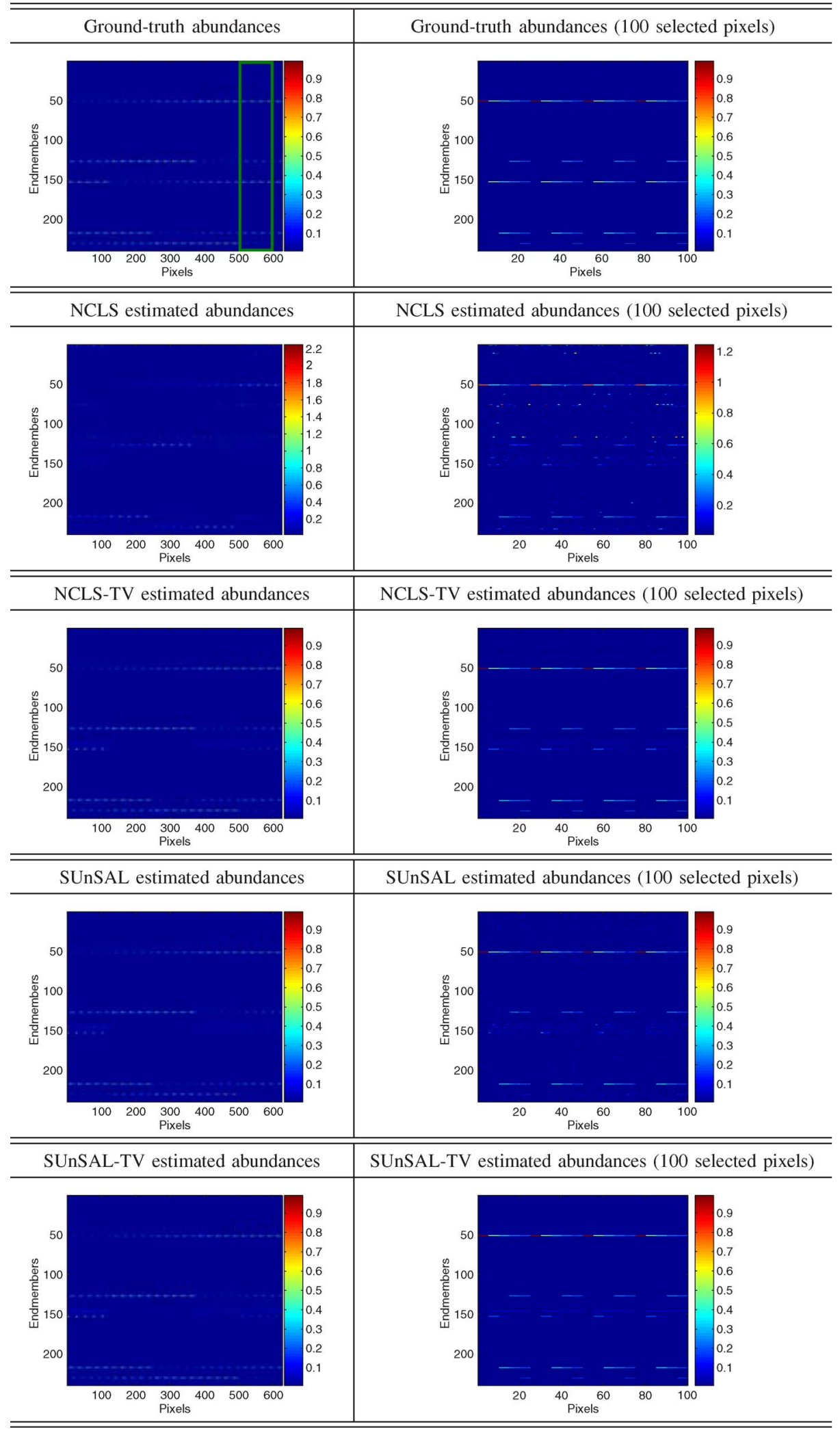

Fig. 7. Ground-truth and estimated abundances obtained for each endmember material in the $\mathbf{A}_{1}$ library in the the scene DC1 affected by white noise, simulated with SNR of $30 \mathrm{~dB}$ (leftmost column), and the same results for 100 selected pixels marked in a green rectangle in the upper-leftmost plot (rightmost column).

estimated for other endmembers exhibited similar behavior, we only report the results observed for endmember \#5 in DC1 (see Fig. 1) and for endmember \#9 in DC2 (see Fig. 2). The abundance maps displayed in Figs. 5 and 6 were obtained using optimal values for parameters $\lambda$ and $\lambda_{T V}$ (see Tables I and II). From Figs. 5 and 6, it can be seen that the spatial term based on the TV regularizer improves both the NCLS and the SUnSAL solutions. In qualitative fashion, we can 
observe that the regions with high fractional abundance of the considered endmember are better delineated, while mixed regions with low concentration of the considered endmember are more homogeneous in nature. No matter the level of noise, the solutions get spatial consistency and the spatial distribution of the materials is determined with good accuracy in both cases. Sparse solutions are particularly needed when the SNR is low, as reported in Tables I and II.

Fig. 7 shows the fractional abundance estimations obtained for each endmember material in the $\mathbf{A}_{1}$ library (as a function of the pixel index in the scene DC1 simulated with SNR of $30 \mathrm{~dB}$ and white noise) by the methods, along with the ground-truth abundances. To facilitate the visualization, background pixels are removed from the plots in the leftmost column of Fig. 7. In order to better visualize the impact of applying the TV regularizer, the rightmost column of Fig. 7 shows the same abundances for only 100 selected pixels (indicated by the green rectangle in the upper-leftmost plot in Fig. 7). The parameters used correspond to the ones reported in Table I.

From Fig. 7, it can be seen graphically that the TV regularizer imposes spatial consistency in the unmixing results. The lines (denoting the abundance of a certain endmember in all pixels of the image) estimated by SUnSAL-TV are more similar to those in the ground-truth than the ones estimated by SUnSAL. After applying SUnSAL, there are many low abundance values estimated for endmembers which are not actually present in the image, but the TV regularizer vanishes those values and provides an overall estimate which is closer to ground-truth values. Note that, although NCLS provides fractional abundances larger than one, the accuracy of the results is clearly improved when the TV regularizer is used. It is also remarkable that, at first sight, it would appear that all the methods encounter problems in accurately locating one endmember (i.e., the one situated around line 150 of the upper-leftmost plot in Fig. 7). However, we must note that the spectral signature of this endmember corresponds to the muscovite mineral, and there are 11 spectra in the library corresponding to different variations of this mineral (all very similar in spectral terms-within $2.52 \circ$ - and located around line 150 of the upper-leftmost plot in Fig. 7). As a result, we conclude that the abundance estimates provided by NCLS-TV and by SUnSAL-TV are highly accurate and indeed correlated with the ground-truth ones. With the aforementioned observations in mind, we believe that the combination of the TV regularizer with sparse unmixing methods offers promising results. Although the results obtained with simulated data sets are quite encouraging and revealing of the potential of including spatial information in sparse unmixing formulations, further experiments should be conducted with real hyperspectral scenes in order to fully substantiate our findings in real analysis scenarios.

To conclude this section, Table III illustrates the per-pixel execution times for the algorithms compared in this section. The values reported correspond to the average times, measured after processing 600 pixels randomly chosen from DC1 and DC2 (300 pixels from each datacube, 100 for each noise level), while the spectral libraries used were the ones shown in Section IV-A. The algorithms were implemented using Matlab7 on a desktop PC equipped with an Intel Core 2 Quad CPU (at $2.33 \mathrm{GHz}$ ) and $4 \mathrm{~GB}$ of RAM memory.
TABLE III

Average Per-Pixel Processing Times (In Seconds) Measured After Applying The Considered UnmiXing Algorithms to 600 Randomly Chosen Pixels From Simulated Images DC1 AND DC2, ON A DESKTOP PC EQUIPPED WITH AN INTEL CORE 2 QUAD CPU (AT $2.33 \mathrm{GHz}$ ) AND 4 GB OF RAM MEMORY

\begin{tabular}{c|cccc}
\hline \hline & NCLS & NCLS-TV & SUnSAL & SUnSAL-TV \\
\hline Time [s] & 0.026 & 1.614 & 0.028 & 1.625 \\
\hline \hline
\end{tabular}

\section{EXPERIMENTS With REAL DATA}

The scene used in our real data experiments is the wellknown AVIRIS Cuprite data set, available online in reflectance units. ${ }^{4}$ This scene has been widely used to validate the performance of endmember extraction algorithms. The portion used in experiments corresponds to a $350 \times 350$-pixel subset of the sector labeled as f970619t01p02_r02_\{s\}c03.a.rfl in the online data. The scene comprises 224 spectral bands between 0.4 and $2.5 \mu \mathrm{m}$, with nominal spectral resolution of $10 \mathrm{~nm}$. Prior to the analysis, bands $1-2,105-115,150-170$, and 223-224 were removed due to water absorption and low SNR in those bands, leaving a total of 188 spectral bands. The Cuprite site is well understood mineralogically and has several exposed minerals of interest, all included in the USGS library considered in experiments, denoted splib06 $6^{5}$ and released in September 2007. In our experiments, we use spectra obtained from this library as input to the unmixing methods described in Section III. For illustrative purposes, Fig. 8 shows a mineral map produced in 1995 by USGS, in which the Tricorder 3.3 software product [69] was used to map different minerals present in the Cuprite mining district ${ }^{6}$. It should be noted that the Tricorder map is only available for hyperspectral data collected in 1995, while the publicly available AVIRIS Cuprite data was collected in 1997. Therefore, a direct comparison between the 1995 USGS map and the 1997 AVIRIS data is not possible. However, the USGS map serves as a good indicator for qualitative assessment of the fractional abundance maps produced by the different unmixing algorithms discussed in Section III.

Before unmixing the AVIRIS Cuprite hyperspectral data, we addressed possible calibration mismatches between the real image spectra and the spectra available in the library. This is because, even though we are working with atmospherically corrected data in reflectance units, these calibration interferers are still present due to the rather different acquisition conditions of the two data types. In order to minimize these mismatches, we applied a band-dependent correction strategy to the original data set, which amounts at replacing the data set $\mathbf{Y}$ with $\mathbf{C Y}$, where $\mathbf{C}$ is a diagonal matrix that minimizes the modeling error, i.e.,

$$
\widehat{\mathbf{C}}=\arg \min _{\mathbf{C}, \mathbf{X} \geq 0, \mathbf{1}_{m}^{T} \mathbf{X}=\mathbf{1}_{n}^{T}}\left\|\mathbf{A}_{1} \mathbf{X}-\mathbf{C Y}\right\|_{2}
$$

where $\mathbf{X} \geq \mathbf{0}$ is the fractional abundance matrix. The problem in (15) is nonconvex and, thus, very hard to solve exactly. In this paper,we have computed a suboptimal solution to this

\footnotetext{
${ }^{4} \mathrm{http}: / /$ aviris.jpl.nasa.gov/html/aviris.freedata.html

${ }^{5} \mathrm{http}: / /$ speclab.cr.usgs.gov/spectral.lib06

${ }^{6} \mathrm{http}: / /$ speclab.cr.usgs.gov/cuprite95.tgif.2.2um_map.gif
} 


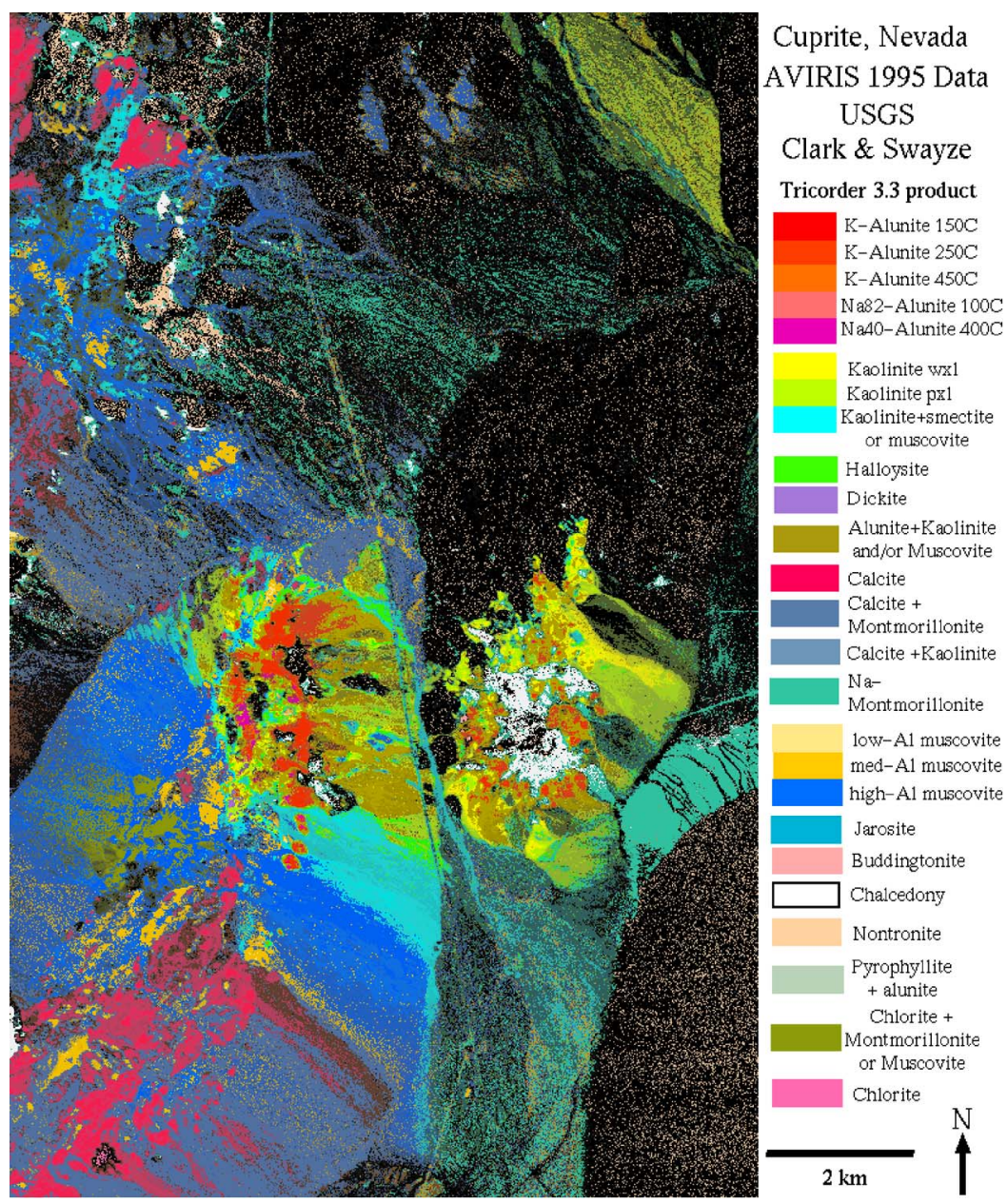

Fig. 8. USGS map showing the location of different minerals in the Cuprite mining district in Nevada. The map is available online at http://speclab.cr.usgs.gov/cuprite95.tgif.2.2um_map.gif.

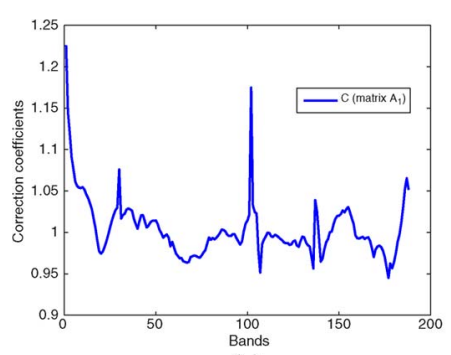

(a)

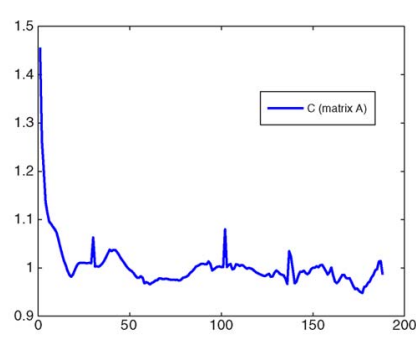

(b)
Fig. 9. Plot of diagonal values of the correction matrix $\mathbf{C}$ for the following spectral libraries: (a) $\mathbf{A}_{1}$, and (b) $\mathbf{A}$.

problem by alternating the minimization with respect to $\mathbf{C}$ and to $\mathbf{X}$ [44]. In our experiments, we used the matrix $\mathbf{A}_{1}$ described in Section IV-A and also a library (denoted by $\mathbf{A}$ ) which contains 498 minerals randomly selected from the USGS library. The correction matrices corresponding to $\mathbf{A}_{1}$ and $\mathbf{A}$ are, respectively displayed in Fig. 9(a) and (b).

Fig. 10 shows a visual (qualitative) comparison between the fractional abundance maps estimated for the minerals: alunite, buddingtonite and chalcedony by applying the NCLS, NCLS-TV, SUnSAL, and SUnSAL-TV algorithms to the AVIRIS Cuprite scene using the library $\mathbf{A}_{1}$. These minerals are known to be present (in prominent fashion) in the Cuprite mining district. For comparative purposes, the spatial distribution maps of these materials extracted from the Tricorder software product in Fig. 8 are also displayed in Fig. 10. On the other hand, Fig. 11 displays the abundance maps estimated for the same minerals using the different unmixing algorithms considered in this work and the spectral library $\mathbf{A}$. The parameters used in all cases (after empirical optimization) were $\lambda=\lambda_{T V}=10^{-3}$ for NCLS-TV, SUnSAL, and SUnSAL-TV. For the NCLS, both $\lambda$, and $\lambda_{T V}$ were set to zero.

From Figs. 10 and 11, it can be observed that the abundance maps obtained by the methods with the TV spatial regularizer exhibit good spatial consistency of minerals of interest and less outliers than the maps without such regularizer. This can be appreciated, for instance, in the buddingtonite maps in both figures. In this particular mineral, the TV term helps both NCLS-TV and SUnSAL-TV to reduce toward zero some outlier values of high abundance of the mineral in isolated regions of the image that can be observed for the NCLS and SUnSAL results. In this case, the TV term allows obtaining a better characterization of the buddingtonite mineral (which appears as an anomaly in the scene) in accordance with the Tricorder 3.3 software product.

Another important observation from Figs. 10 and 11 is that the results obtained by sparse unmixing methods using $\mathbf{A}_{1}$ and $\mathbf{A}$ are very similar, indicating that sparse unmixing is quite insensitive to the number of spectra in the reference library, and regardless of the inclusion of the TV term in the solution of the sparse unmixing problem. Although the effect of the TV 

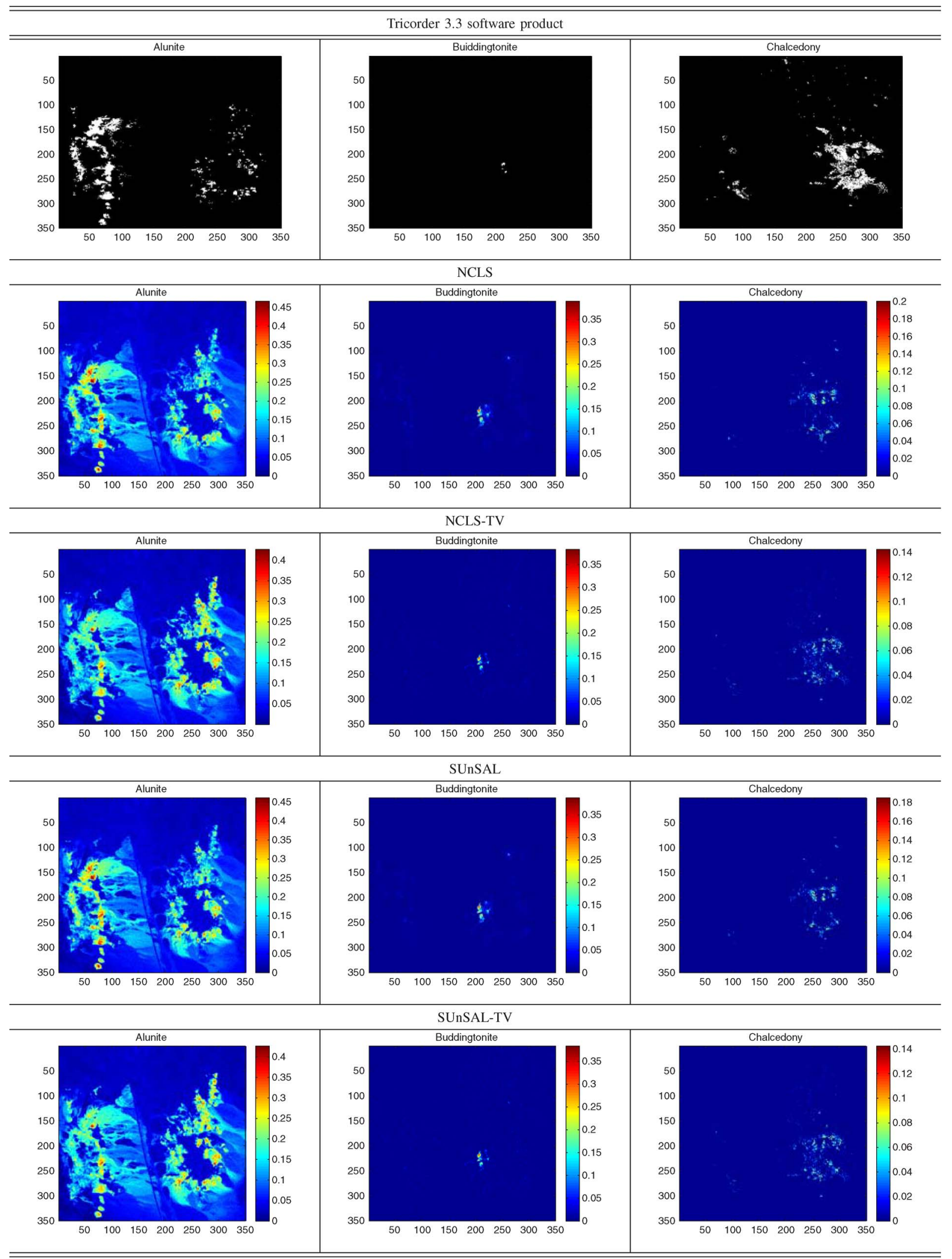

Fig. 10. Abundance maps estimated for the minerals: alunite, buddingtonite, and chalcedony by applying the NCLS, NCLS-TV, SUnSAL and SUnSAL-TV algorithms to the AVIRIS Cuprite scene using the library $\mathbf{A}_{1}$.

regularizer is not as apparent in the real image experiments as in the simulated image experiments (which is probably due to the fact that the AVIRIS Cuprite image used in experiments is characterized by high SNR), the behavior observed is in line with the results obtained for the experiments carried out on simulated data, indicating that the inclusion of spatial 

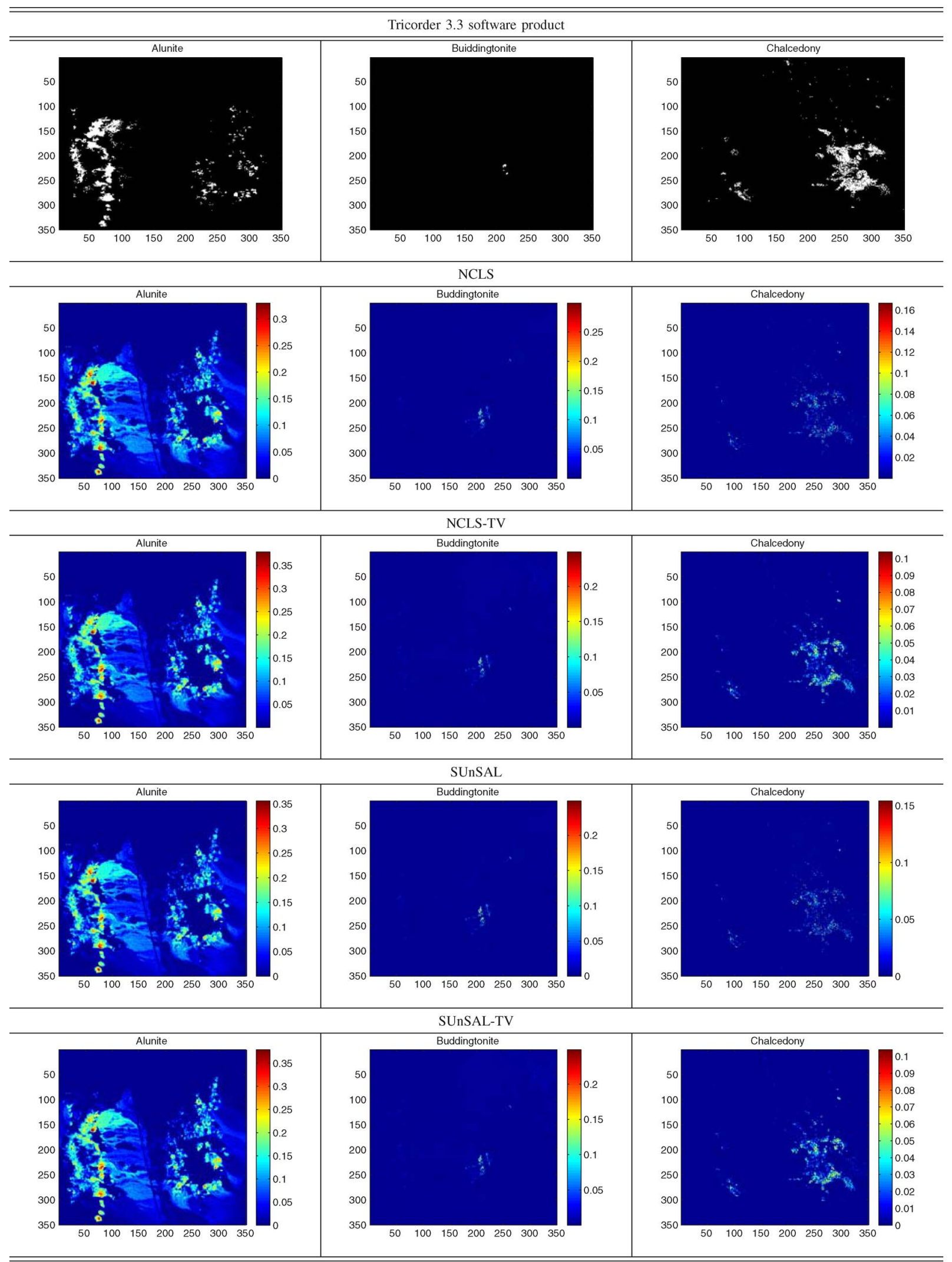

Fig. 11. Abundance maps estimated for the minerals: alunite, buddingtonite, and chalcedony by applying the NCLS, NCLS-TV, SUnSAL, and SUnSAL-TV algorithms to the AVIRIS Cuprite scene using the library A.

information in sparse unmixing by means of the proposed TV regularizer can assist in obtaining piecewise smooth abundance maps with less outliers and high spatial consistency. Further experiments with additional hyperspectral scenes and libraries should be conducted in future work in order to fully objectify our findings. 


\section{CONCLUSiOn AND Future Work}

Sparse unmixing has been recently introduced as a powerful methodology to solve the spectral unmixing problem by taking advantage of spectral libraries of materials measured on the ground, thus reducing the dependency of unmixing methods on the availability of pure spectral signatures in the input data or on the ability of an endmember extraction algorithm to identify the number and spectra of such pure signatures. Despite the sucess of sparse unmixing in different applications, a limitation of this approach has been the fact that it does not consider the spatial correlation between the image features in the unmixing process. To circumvent this limitation, in this work, we have revisited the sparse unmixing formulation and introduced a TV regularizer term aimed at imposing spatial consistency in hyperspectral sparse unmixing solutions. A new algorithm, called sparse unmixing via variable splitting augmented Lagrangian and TV (SUnSAL-TV) has been specifically developed for this purpose. Our experimental results (conducted using both simulated and real hyperspectral data sets) indicate that the inclusion of the TV regularizer offers the potential to improve unmixing performance in two different analysis scenarios, i.e., when the sparsity is imposed explicitly (SUnSAL), and also when it is not enforced (NCLS). This is particularly the case for scenarios with high noise levels. In this way, the limitations of sparse unmixing approaches related with the high coherence of spectral signatures or the lack of spatial-contextual information when solving the unmixing problem have been significantly mitigated. Our future work will focus on a full evaluation of isotropic versus nonisotropic TV regularization, defined in the spatial dimension, and also on the development of fast parallel algorithms able to cope with the high computational complexity of the proposed approach. It should be noted that this issue is subject to further improvements related with the inherently parallel nature of sparse unmixing algorithms combined with the TV regularizer, which are expected to scale linearly on parallel systems such as multicore processors or graphics processing units. Another relevant topic deserving further research is a detailed evaluation of the influence of neighborhood systems in the proposed approach. In this paper, we only used first-order neighborhood systems in the definition of the TV regularizer. However, second-order neighborhood systems may be well-suited for piecewise affine surfaces.

\section{APPENDIX}

In this appendix, we detail the SUnSAL-TV algorithm introduced in Section III. We start by expanding the augmented Lagrangian introduced in (14):

$$
\begin{aligned}
\mathcal{L}(\mathbf{U}, & \left.\mathbf{V}_{1}, \mathbf{V}_{2}, \mathbf{V}_{3}, \mathbf{V}_{4}, \mathbf{V}_{5}, \mathbf{D}_{1}, \mathbf{D}_{2}, \mathbf{D}_{3}, \mathbf{D}_{4}, \mathbf{D}_{5}\right) \\
= & \frac{1}{2}\left\|\mathbf{V}_{1}-\mathbf{Y}\right\|_{F}^{2}+\lambda\left\|\mathbf{V}_{2}\right\|_{1,1}+\lambda_{T V}\left\|\mathbf{V}_{4}\right\|_{1,1}+\iota_{R+}\left(\mathbf{V}_{5}\right) \\
& +\frac{\mu}{2}\left\|\mathbf{A} \mathbf{U}-\mathbf{V}_{1}-\mathbf{D}_{1}\right\|_{F}^{2}+\frac{\mu}{2}\left\|\mathbf{U}-\mathbf{V}_{2}-\mathbf{D}_{2}\right\|_{F}^{2} \\
& +\frac{\mu}{2}\left\|\mathbf{U}-\mathbf{V}_{3}-\mathbf{D}_{3}\right\|_{F}^{2}+\frac{\mu}{2}\left\|\mathbf{H} \mathbf{V}_{3}-\mathbf{V}_{4}-\mathbf{D}_{4}\right\|_{F}^{2} \\
& +\frac{\mu}{2}\left\|\mathbf{U}-\mathbf{V}_{5}-\mathbf{D}_{5}\right\|_{F}^{2} .
\end{aligned}
$$

The pseudocode of SUnSAL-TV is shown in Algorithm 2. It is the expansion of the ADDM algorithm presented in Algorithm 1, with a single optimization with respect to $\mathbf{V}_{3}$ and with respect to $\mathbf{V}_{4}$, per iteration.

Algorithm 2 Pseudocode of the SUnSAL-TV algorithm.

1. Initialization: set $k=0$, choose $\mu \geq 0, \mathbf{U}^{(0)}, \mathbf{V}_{1}^{(0)}, \ldots$, $\mathbf{V}_{5}^{(0)}, \mathbf{D}_{1}^{(0)}, \ldots, \mathbf{D}_{5}^{(0)}$

\section{2. repeat:}

3. $\mathbf{U}^{(k+1)} \leftarrow \arg \min _{\mathbf{U}} \mathcal{L}\left(\mathbf{U}, \mathbf{V}_{1}^{(k)}, \ldots, \mathbf{V}_{5}^{(k)}, \mathbf{D}_{1}^{(k)}, \ldots\right.$, $\left.\mathbf{D}_{5}^{(k)}\right)$

4. for $i=1, \ldots 5$

5. $\quad \mathbf{V}_{i}^{(k+1)} \leftarrow \arg \min _{\mathbf{V}_{i}} \mathcal{L}\left(\mathbf{U}^{(k)}, \mathbf{V}_{1}^{(k)}, \ldots, \mathbf{V}_{i}, \ldots, \mathbf{V}_{5}^{(k)}\right)$

6. end for

7. Update Lagrange multipliers:

$$
\begin{aligned}
& \mathbf{D}_{1}^{(k+1)} \leftarrow \mathbf{D}_{1}^{(k)}-\mathbf{A} \mathbf{U}^{(k+1)}+\mathbf{V}_{1}^{(k+1)} \\
& \mathbf{D}_{2}^{(k+1)} \leftarrow \mathbf{D}_{2}^{(k)}-\mathbf{U}^{(k+1)}+\mathbf{V}_{2}^{(k+1)} \\
& \mathbf{D}_{3}^{(k+1)} \leftarrow \mathbf{D}_{3}^{(k)}-\mathbf{U}^{(k+1)}+\mathbf{V}_{3}^{(k+1)} \\
& \mathbf{D}_{4}^{(k+1)} \leftarrow \mathbf{D}_{4}^{(k)}-\mathbf{H} \mathbf{V}_{3}^{(k+1)}+\mathbf{V}_{4}^{(k+1)} \\
& \mathbf{D}_{5}^{(k+1)} \leftarrow \mathbf{D}_{5}^{(k)}-\mathbf{U}^{(k+1)}+\mathbf{V}_{5}^{(k+1)}
\end{aligned}
$$

8. Update iteration: $k \leftarrow k+1$

9. until some stopping criterion is satisfied.

We give now details about step 3 of Algorithm 2. The goal of this step is to determine the value of the variable $\mathbf{U}$ at each iteration. Given that we run an optimization over the variable $\mathbf{U}$, the terms of the objective function (11) which do no contain this variable are not taken into account. The reduced optimization function becomes, then

$$
\begin{aligned}
& \mathbf{U}^{(k+1)} \leftarrow \arg \min _{\mathbf{U}} \frac{\mu}{2}\left\|\mathbf{A} \mathbf{U}-\mathbf{V}_{1}^{(k)}-\mathbf{D}_{1}^{(k)}\right\|_{F}^{2} \\
& +\frac{\mu}{2}\left\|\mathbf{U}-\mathbf{V}_{2}^{(k)}-\mathbf{D}_{2}^{(k)}\right\|_{F}^{2}+\frac{\mu}{2}\left\|\mathbf{U}-\mathbf{V}_{3}^{(k)}-\mathbf{D}_{3}^{(k)}\right\|_{F}^{2} \\
& \quad++\frac{\mu}{2}\left\|\mathbf{U}-\mathbf{V}_{5}^{(k)}-\mathbf{D}_{5}^{(k)}\right\|_{F}^{2} .
\end{aligned}
$$

The solution of (17) is simply

$$
\mathbf{U}^{(k+1)} \leftarrow\left(\mathbf{A}^{T} \mathbf{A}+3 \mathbf{I}\right)^{-1}\left(\mathbf{A}^{T} \xi_{1}+\xi_{2}+\xi_{4}+\xi_{5}\right)
$$

where $\mathbf{I}$ is the identity matrix, $\mathbf{A}^{T}$ represents the transpose of $\mathbf{A}$ and: $\xi_{1}=\mathbf{V}_{1}^{(k)}+\mathbf{D}_{1}^{(k)}, \xi_{2}=\mathbf{V}_{2}^{(k)}+\mathbf{D}_{2}^{(k)}, \xi_{3}=\mathbf{V}_{3}^{(k)}+$ $\mathbf{D}_{3}^{(k)}, \xi_{5}=\mathbf{V}_{5}^{(k)}+\mathbf{D}_{5}^{(k)}$. Notice that matrix $\mathbf{A}$ acts only on the spectral domain.

On the other hand, step 5 of SUnSAL-TV computes the values of the variables $\mathbf{V}_{1}, \ldots, \mathbf{V}_{5}$ at the current iteration. To compute $\mathbf{V}_{1}$, the optimization problem to be solved is

$\mathbf{V}_{1}^{(k+1)} \leftarrow \arg \min _{\mathbf{V}_{1}} \frac{1}{2}\left\|\mathbf{V}_{1}-\mathbf{Y}\right\|_{F}^{2}+\frac{\mu}{2}\left\|\mathbf{A} \mathbf{U}^{(k)}-\mathbf{V}_{1}-\mathbf{D}_{1}^{(k)}\right\|_{F}^{2}$

whose solution is 


$$
\mathbf{V}_{1}^{(k+1)} \leftarrow \frac{1}{1+\mu}\left[\mathbf{Y}+\mu\left(\mathbf{A} \mathbf{U}^{(k)}-\mathbf{D}_{1}^{(k)}\right)\right]
$$

To compute $\mathbf{V}_{2}$, the optimization problem to be solved is

$$
\mathbf{V}_{2}^{(k+1)} \leftarrow \arg \min _{\mathbf{V}_{2}} \lambda\left\|\mathbf{V}_{2}\right\|_{1,1}+\frac{\mu}{2}\left\|\mathbf{U}^{(k)}-\mathbf{V}_{2}-\mathbf{D}_{2}^{(k)}\right\|_{F}^{2}
$$

whose solution is the well-known soft threshold [70]

$$
\mathbf{V}_{2}^{(k+1)} \leftarrow \operatorname{soft}\left(\xi_{2}, \frac{\lambda}{\mu}\right)
$$

where $\xi_{2}=\mathbf{V}_{2}^{(k)}+\mathbf{D}_{2}^{(k)} \quad$ and $\operatorname{soft}(\cdot, \tau)$ denotes the component-wise application of the soft-threshold function $y \mapsto \operatorname{sign}(y) \max \{|y|-\tau, 0\}$.

In order to compute $\mathbf{V}_{3}$, we solve the optimization problem

$$
\begin{aligned}
\mathbf{V}_{3}^{(k+1)} \leftarrow \arg \min _{\mathbf{V}_{3}} \frac{\mu}{2} \| & \mathbf{U}^{(k)}-\mathbf{V}_{3}-\mathbf{D}_{3}^{(k)} \|_{F}^{2} \\
& +\frac{\mu}{2}\left\|\mathbf{H} \mathbf{V}_{3}-\mathbf{V}_{4}^{(k)}-\mathbf{D}_{4}^{(k)}\right\|_{F}^{2}
\end{aligned}
$$

having the solution

$$
\mathbf{V}_{3}^{(k+1)} \leftarrow\left(\mathbf{H}^{T} \mathbf{H}+\mathbf{I}\right)^{-1}\left(\mathbf{U}^{(k)}-\mathbf{D}_{3}^{(k)}+\mathbf{H}^{T} \xi_{4}\right)
$$

where $\xi_{4}=\mathbf{V}_{4}^{(k)}+\mathbf{D}_{4}^{(k)}$. Note that $\mathbf{H}^{T} \mathbf{H}+\mathbf{I}$ acts only on the spatial domain and can be applied independently, in band-byband fashion. Since, for each band, $\mathbf{H}$ is a convolution, it can be computed efficiently using the discrete Fourier transform diagonalization.

$\mathbf{V}_{4}$ is computed by solving the optimization problem

$$
\mathbf{V}_{4}^{(k+1)} \leftarrow \arg \min _{\mathbf{V}_{4}} \lambda_{T V}\left\|\mathbf{V}_{4}\right\|_{1,1}+\frac{\mu}{2}\left\|\mathbf{H} \mathbf{V}_{3}^{(k)}-\mathbf{V}_{4}-\mathbf{D}_{4}^{(k)}\right\|_{F}^{2}
$$

As for (21), the solution of (25) is the soft threshold

$$
\mathbf{V}_{4}^{(k+1)} \leftarrow \operatorname{soft}\left(\mathbf{D}_{4}^{(k)}-\mathbf{H} \mathbf{V}_{3}^{(k)}, \frac{\lambda_{T V}}{\mu}\right)
$$

Finally, $\mathbf{V}_{5}$ is computed by solving the following optimization problem:

$$
\mathbf{V}_{5}^{(k+1)} \leftarrow \arg \min _{\mathbf{V}_{5}} \iota_{R+}\left(\mathbf{V}_{5}\right)+\frac{\mu}{2}\left\|\mathbf{U}^{(k)}-\mathbf{V}_{5}-\mathbf{D}_{5}^{(k)}\right\|_{F}^{2} .
$$

In (27), the role of the $\iota_{R+}$ term is to project the solution onto the nonnegative orthant and the value of $\mathbf{V}_{5}$ is given by

$$
\mathbf{V}_{5}^{(k+1)} \leftarrow \max \left(\mathbf{U}^{(k)}-\mathbf{D}_{5}^{(k)}, 0\right) .
$$

Concerning the computational complexity of the SUnSALTV algorithm, the most costly steps are the calculus of $\mathbf{U}$, which has the order of complexity $\mathcal{O}\left(n L^{2}\right)$, and of $\mathbf{V}_{3}$, with the order $\mathcal{O}(\operatorname{Ln} \log n)$, while the others have computational complexity $\mathcal{O}(n)$, where $L$ is the number of spectral bands and $n$ is the number of pixels in the image. The overall order of complexity per iteration is given then by $(n L \cdot \max \{L, \log n\})$. It should be noted that, in real images, $L$ is very likely to be higher than $\log n$ leading to complexity of the order $\mathcal{O}\left(n L^{2}\right)$ for the SUnSAL-TV algorithm.

\section{ACKNOWLEDGMENT}

The authors thank Dr. R. O. Green and Dr. R. N. Clark for, respectively, sharing the AVIRIS Cuprite data and the USGS spectral library with the scientific community. Last but not least, the authors gratefully thank the Associate Editor and the three anonymous reviewers for their outstanding comments and suggestions, which greatly improved the quality and presentation of this manuscript.

\section{REFERENCES}

[1] J. Adams, M. Smith, and P. Johnson, "Spectral mixture modeling: A new analysis of rock and soil types at the Viking Lander 1 site," J. Geophys. Res., vol. 91, pp. 8098-8112, Jul. 1986.

[2] J. Settle and N. Drake, "Linear mixing and the estimation of ground cover proportions," Int. J. Remote Sens., vol. 14, no. 6, pp. 1159-1177, Apr. 1993.

[3] A. Plaza, P. Martinez, R. Perez, and J. Plaza, "A quantitative and comparative analysis of endmember extraction algorithms from hyperspectral data," IEEE Trans. Geosci. Remote Sens., vol. 42, no. 3, pp. 650-663, Mar. 2004.

[4] Q. Du, N. Raksuntorn, N. Younan, and R. King, "End-member extraction for hyperspectral image analysis," Appl. Opt., vol. 47, no. 28, pp. 77-84, Jul. 2008.

[5] D. Heinz and C.-I. Chang, "Fully constrained least squares linear mixture analysis for material quantification in hyperspectral imagery," IEEE Trans. Geosci. Remote Sens., vol. 39, no. 3, pp. 529-545, Mar. 2001.

[6] C.-I. Chang and D. Heinz, "Constrained subpixel target detection for remotely sensed imagery," IEEE Trans. Geosci. Remote Sens., vol. 38, no. 3, pp. 1144-1159, May 2000.

[7] C.-I. Chang, Hyperspectral Imaging: Techniques for Spectral Detection and Classification. New York: Kluwer/Plenum, 2003.

[8] C. Borel and S. Gerstl, "Nonlinear spectral mixing model for vegetative and soil surfaces," Remote Sens. Environ., vol. 47, no. 3, pp. 403-416, Mar. 1994.

[9] W. Liu and E. Wu, "Comparison of non-linear mixture models," Remote Sens. Environ., vol. 18, pp. 1976-2003, 2004.

[10] N. Raksuntorn and Q. Du, "Nonlinear spectral mixture analysis for hyperspectral imagery in an unknown environment," IEEE Geosci. Remote Sens. Lett., vol. 7, no. 4, pp. 836-840, Oct. 2010.

[11] A. Plaza, G. Martin, J. Plaza, M. Zortea, and S. Sanchez, "Recent developments in spectral unmixing and endmember extraction," in Optical Remote Sensing, S. Prasad, L. M. Bruce, and J. Chanussot, Eds. Berlin, Germany: Springer-Verlag, 2011, ch. 12, pp. 235-267.

[12] J. Harsanyi and C.-I. Chang, "Hyperspectral image classification and dimensionality reduction: An orthogonal subspace projection," IEEE Trans. Geosci. Remote Sens., vol. 32, no. 4, pp. 779-785, Jul. 1994.

[13] J. Boardman, F. Kruse, and R. Green, "Mapping target signatures via partial unmixing of AVIRIS data," in Proc. JPL Airborne Earth Sci. Workshop, 1995, pp. 23-26.

[14] J. Bowles, P. Palmadesso, J. Antoniades, M. Baumback, and L. Rickard, "Use of filter vectors in hyperspectral data analysis," in Proc. SPIE Infrared Spaceborne Remote Sens. III, 1995, vol. 2553, pp. 148-157.

[15] R. Neville, K. Staenz, T. Szeredi, J. Lefebvre, and P. Hauff, "Automatic endmember extraction from hyperspectral data for mineral exploration," in Proc. 21 st Can. Symp. Remote Sens., 1999, pp. 21-24.

[16] A. Ifarraguerri and C.-I. Chang, "Multispectral and hyperspectral image analysis with convex cones," IEEE Trans. Geosci. Remote Sens., vol. 37, no. 2, pp. 756-770, Mar. 1999.

[17] M. Winter, "N-FINDR: An algorithm for fast autonomous spectral endmember determination in hyperspectral data," in Proc. SPIE, 1999, vol. 3753, pp. 266-277.

[18] Q. Du, H. Ren, and C.-I. Chang, "A comparative study for orthogonal subspace projection and constrained energy minimization," IEEE Trans. Geosci. Remote Sens., vol. 41, no. 6, pp. 1525-1529, Jun. 2003.

[19] M. Berman, H. Kiiveri, R. Lagerstrom, A. Ernst, R. Dunne, and J. Huntington, "ICE: A statistical approach to identifying endmembers in hyperspectral images," IEEE Trans. Geosci. Remote Sens., vol. 42, no. 10, pp. 2085-2095, Oct. 2004.

[20] J. Nascimento and J. Bioucas-Dias, "Vertex component analysis: A fast algorithm to unmix hyperspectral data," IEEE Trans. Geosci. Remote Sens., vol. 43, no. 4, pp. 898-910, Apr. 2005.

[21] A. Plaza and C.-I. Chang, "Impact of initialization on design of endmember extraction algorithms," IEEE Trans. Geosci. Remote Sens., vol. 44, no. 11, pp. 3397-3407, Nov. 2006. 
[22] C.-I. Chang and A. Plaza, "A fast iterative algorithm for implementation of pixel purity index," IEEE Geosci. Remote Sens. Lett., vol. 3, no. 1, pp. 63-67, Jan. 2006.

[23] D. Rogge, B. Rivard, J. Zhang, and J. Feng, "Iterative spectral unmixing for optimizing per-pixel endmember sets," IEEE Trans. Geosci. Remote Sens., vol. 44, no. 12, pp. 3725-3736, Dec. 2006.

[24] J. Wang and C.-I. Chang, "Applications of independent component analysis in endmember extraction and abundance quantification for hyperspectral imagery," IEEE Trans. Geosci. Remote Sens., vol. 44, no. 9, pp. 2601-2616, Sep. 2006.

[25] C.-I. Chang, C.-C. Wu, W. Liu, and Y.-C. Ouyang, "A new growing method for simplex-based endmember extraction algorithm," IEEE Trans. Geosci. Remote Sens., vol. 44, no. 10, pp. 2804-2819, Oct. 2006.

[26] A. Zare and P. Gader, "Sparsity promoting iterated constrained endmember detection for hyperspectral imagery," IEEE Geosci. Remote Sens. Lett., vol. 4, no. 3, pp. 446-450, Jul. 2007.

[27] A. Zare and P. Gader, "Hyperspectral band selection and endmember detection using sparsity promoting priors," IEEE Geosci. Remote Sens. Lett., vol. 5, no. 2, pp. 256-260, Apr. 2008.

[28] M. Zortea and A. Plaza, "A quantitative and comparative analysis of different implementations of N-FINDR: A fast endmember extraction algorithm," IEEE Geosci. Remote Sens. Lett., vol. 6, no. 4, pp. 787-791, Oct. 2009.

[29] X. Tao, B. Wang, and L. Zhang, "Orthogonal bases approach for the decomposition of mixed pixels in hyperspectral imagery," IEEE Geosci. Remote Sens. Lett., vol. 6, no. 2, pp. 219-223, Apr. 2009.

[30] A. Zare and P. Gader, "PCE: Piecewise convex endmember detection," IEEE Trans. Geosci. Remote Sens., vol. 48, no. 6, pp. 2620-2632, Jun. 2010.

[31] C.-I. Chang, C.-C. Wu, C.-S. Lo, and M.-L. Chang, "Real-time simplex growing algorithms for hyperspectral endmember extraction," IEEE Trans. Geosci. Remote Sens., vol. 48, no. 4, pp. 1834-1850, Apr. 2010.

[32] F. Schmidt, A. Schmidt, E. Treandguier, M. Guiheneuf, S. Moussaoui, and N. Dobigeon, "Implementation strategies for hyperspectral unmixing using Bayesian source separation," IEEE Trans. Geosci. Remote Sens., vol. 48, no. 11, pp. 4003-4013, Nov. 2010.

[33] O. Duran and M. Petrou, "Robust endmember extraction in the presence of anomalies," IEEE Trans. Geosci. Remote Sens., vol. 49, no. 6, pp. 19861996, Jun. 2011.

[34] B. Zhang, X. Sun, L. Gao, and L. Yang, "Endmember extraction of hyperspectral remote sensing images based on the ant colony optimization (ACO) algorithm," IEEE Trans. Geosci. Remote Sens., vol. 49, no. 7, pp. 2635-2646, Jul. 2011.

[35] M. Shoshany, F. Kizel, N. Netanyahu, N. Goldshlager, T. Jarmer, and G. Even-Tzur, "An iterative search in end-member fraction space for spectral unmixing," IEEE Geosci. Remote Sens. Lett., vol. 8, no. 4, pp. 706-709, Jul. 2011.

[36] C.-I. Chang, C.-C. Wu, and H.-M. Chen, "Random pixel purity index," IEEE Geosci. Remote Sens. Lett., vol. 7, no. 2, pp. 324-328, Apr. 2010.

[37] S. Dowler and M. Andrews, "On the convergence of N-FINDR and related algorithms: To iterate or not to iterate?" IEEE Geosci. Remote Sens. Lett., vol. 8, no. 1, pp. 4-8, Jan. 2011.

[38] M. Craig, "Minimum-volume transforms for remotely sensed data," IEEE Trans. Geosci. Remote Sens., vol. 32, no. 3, pp. 542-552, May 1994.

[39] L. Miao and H. Qi, "Endmember extraction from highly mixed data using minimum volume constrained nonnegative matrix factorization," IEEE Trans. Geosci. Remote Sens., vol. 45, no. 3, pp. 765-777, Mar. 2007.

[40] J. Li and J. Bioucas-Dias, "Minimum volume simplex analysis: A fast algorithm to unmix hyperspectral data," in Proc. IEEE Int. Geosci. Remote Sens. Symp., 2008, vol. 3, pp. 250-253.

[41] T.-H. Chan, C.-Y. Chi, Y.-M. Huang, and W.-K. Ma, "A convex analysisbased minimum-volume enclosing simplex algorithm for hyperspectral unmixing," IEEE Trans. Signal Process., vol. 57, no. 11, pp. 4418-4432, Nov. 2009.

[42] J. Bioucas-Dias and J. Nascimento, "Hyperspectral unmixing based on mixtures of Dirichlet components," IEEE Trans. Geosci. Remote Sens., vol. 50, no. 3, pp. 863-878, Mar. 2012.

[43] X. Chen, J. Chen, X. Jia, B. Somers, J. Wu, and P. Coppin, "A quantitative analysis of virtual endmembers' increased impact on the collinearity effect in spectral unmixing," IEEE Trans. Geosci. Remote Sens., vol. 49, no. 8, pp. 2945-2956, Aug. 2011.

[44] D. Iordache, J. Bioucas-Dias, and A. Plaza, "Sparse unmixing of hyperspectral data," IEEE Trans. Geosci. Remote Sens., vol. 49, no. 6, pp. 2014 2039, Jun. 2011.

[45] J. Bioucas-Dias and M. Figueiredo, "Alternating direction algorithms for constrained sparse regression: Application to hyperspectral unmixing," in Proc. 2nd Workshop Hyperspectr. Image Signal Process. -Evol. Remote Sens., 2010, vol. 1, pp. 1-4.
[46] J. Eckstein and D. Bertsekas, "On the Douglas-Rachford splitting method and the proximal point algorithm for maximal monotone operators," Math. Program. Ser. A/B, vol. 55, no. 3, pp. 293-318, Jun. 1992.

[47] M. Afonso, J. Bioucas-Dias, and M. Figueiredo, "A fast algorithm for the constrained formulation of compressive image reconstruction and other linear inverse problems," in Proc. IEEE Int. Conf. Acoust., Speech, Signal Process., 2010, vol. 1, pp. 4034-4037.

[48] M. Figueiredo, J. Bioucas-Dias, and M. Afonso, "Fast frame-based image deconvolution using variable splitting and constrained optimization," in Proc. IEEE Workshop Stat. Signal Process., 2009, vol. 1, pp. 109-112.

[49] A. Castrodad, Z. Xing, J. Greer, E. Bosch, L. Carin, and G. Sapiro, "Learning discriminative sparse representations for modeling, source separation, and mapping of hyperspectral imagery," IEEE Trans. Geosci. Remote Sens., vol. 49, no. 11, pp. 4263-4281, Nov. 2011.

[50] A. Charles, B. Olshausen, and C. Rozell, "Learning sparse codes for hyperspectral imagery," IEEE J. Sel. Topics Signal Process., vol. 5, no. 5, pp. 963-978, Sep. 2011.

[51] Y. Qian, S. Jia, J. Zhou, and A. Robles-Kelly, "Hyperspectral unmixing via $L_{1} / 2$ sparsity-constrained nonnegative matrix factorization," IEEE Trans. Geosci. Remote Sens., vol. 49, no. 11, pp. 4282-4297, Nov. 2011.

[52] A. Huck, M. Guillaume, and J. Blanc-Talon, "Minimum dispersion constrained nonnegative matrix factorization to unmix hyperspectral data," IEEE Trans. Geosci. Remote Sens., vol. 48, no. 6, pp. 2590-2602, Jun. 2010.

[53] X. Liu, W. Xia, B. Wang, and L. Zhang, "An approach based on constrained nonnegative matrix factorization to unmix hyperspectral data," IEEE Trans. Geosci. Remote Sens., vol. 49, no. 2, pp. 757-772, Feb. 2011.

[54] M. Zortea and A. Plaza, "Spatial preprocessing for endmember extraction," IEEE Trans. Geosci. Remote Sens., vol. 47, no. 8, pp. 2679-2693, Aug. 2009.

[55] A. Plaza, P. Martinez, R. Perez, and J. Plaza, "Spatial/spectral endmember extraction by multidimensional morphological operations," IEEE Trans. Geosci. Remote Sens., vol. 40, no. 9, pp. 2025-2041, Sep. 2002.

[56] D. Rogge, B. Rivard, J. Zhang, A. Sanchez, J. Harris, and J. Feng, "Integration of spatial-spectral information for the improved extraction of endmembers,"Remote Sens. Environ., vol. 110, no. 3, pp. 287-303, Oct. 2007.

[57] A. Bruckstein, M. Elad, and M. Zibulevsky, "On the uniqueness of nonnegative sparse solutions to underdetermined systems of equations," IEEE Trans. Inf. Theory, vol. 54, no. 11, pp. 4813-4820, Nov. 2008.

[58] E. Candès and T. Tao, "Decoding by linear programming," IEEE Trans. Inf. Theory, vol. 51, no. 12, pp. 4203-4215, Dec. 2005.

[59] L. Rudin, S. Osher, and E. Fatemi, "Nonlinear total variation based noise removal algorithms," Phys. D, Nonlinear Phenom., vol. 60, no. 1-4, pp. 259-268, Nov. 1992.

[60] Z. Guo, T. Wittman, and S. Osher, "L1 unmixing and its application to hyperspectral image enhancement," in Proc. SPIE Conf. Algorithms Technol. Multispectr. Hyperspectr., Ultraspectr. Imagery XV, Orlando, FL, 2009, vol. 7334, pp. 73 341-73449.

[61] A. Chambolle, "An algorithm for total variation minimization and applications," J. Math. Imag. Vis., vol. 20, no. 1/2, pp. 89-97, Jan.-Mar. 2004.

[62] M. Afonso, J. Bioucas-Dias, and M. Figueiredo, "An augmented Lagrangian approach to the constrained optimization formulation of imaging inverse problems," IEEE Trans. Image Process., vol. 20, no. 3, pp. 681-695, Mar. 2011.

[63] A. Zymnis, S. Kim, J. Skaf, M. Parente, and S. Boyd, "Hyperspectral image unmixing via alternating projected subgradients," in Proc. Asilomar Conf. Signals, Syst., Comput., Nov. 2007, pp. 1164-1168.

[64] S. Chen, D. Donoho, and M. Saunders, "Atomic decomposition by basis pursuit," SIAM Rev., vol. 43, no. 1, pp. 129-159, Jan. 2001.

[65] E. Esser, "Applications of Lagrangian-based alternating direction methods and connections to Split-Bregman," Univ. California, Berkeley, CA, Tech. Rep. 09-31, 2009.

[66] B. He and S. Wang, "Alternating direction method with self-adaptive penalty parameters for monotone variational inequalities," J. Optim. Theory Appl., vol. 106, no. 2, pp. 337-356, Aug. 2000.

[67] S. L. Wang and L. Z. Liao, "Decomposition method with a variable parameter for a class of monotone variational inequality problems," J. Optim. Theory Appl., vol. 102, no. 2, pp. 415-429, May 2001.

[68] S. Boyd, N. Parikh, E. Chu, B. Peleato, and J. Eckstein, "Distributed optimization and statistical learning via the alternating direction method of multipliers," Found. Trends Mach. Learn., vol. 3, no. 1, pp. 1-122, 2011.

[69] R. Clark, G. Swayze, K. Livo, R. Kokaly, S. Sutley, J. Dalton, R. McDougal, and C. Gent, "Imaging spectroscopy: Earth and planetary remote sensing with the USGS Tetracorder and expert systems," J. Geophys. Res., vol. 108, no. E12, p. 5131, Dec. 2003.

[70] P. Combettes and V. Wajs, "Signal recovery by proximal forwardbackward splitting," SIAM J. Multiscale Model. Simul., vol. 4, no. 4, pp. $1168-1200,2005$ 


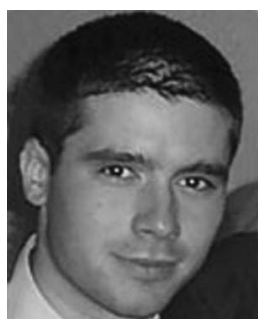

Marian-Daniel Iordache received the M.Sc. degree from the Faculty of Electrical Engineering, Politehnica University of Bucharest, Bucharest, Romania, in July 2006, and the Ph.D. degree in electrical and computer engineering from Instituto Superior Tecnico, Lisbon, Portugal, in December 2011.

His research activity started in 2006 at the Electrical Engineering Research Center, Bucharest, where he worked on several national and European projects dedicated to microelectromechanical systems and high-frequency circuits. From 2008 to 2011, he was a Marie Curie Fellow with the Hyperspectral Imaging Network project funded by the European Commission under the Sixth Framework Programme. Since 2010, he is a member of the Hyperspectral Computing Laboratory research group, University of Extremadura, Caceres. Spain. For his research activity and social implication, he was awarded with the prize The Romanian Student of the Year in Europe 2011, offered by the League of Romanian Students Abroad. He is currently carrying out his research as a post-doc researcher at the Flemish Institute for Technological Research, Center for Remote Sensing and Earth Observation Processes (TAP), Mol, Belgium. His research is focused on hyperspectral unmixing, with emphasis on the sparse characteristics of the solutions.

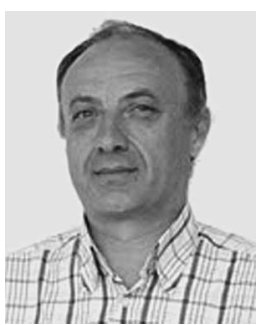

José M. Bioucas-Dias (S'87-M'95) received the E.E., M.Sc., Ph.D., and "Agregado" degrees in electrical and computer engineering, all from Instituto Superior Técnico (IST), the engineering school of the Technical University of Lisbon, Lisbon, Portugal, in 1985, 1991, 1995, and 2007, respectively.

Since 1985, he has been with the Department of Electrical and Computer Engineering, IST. He is also a Senior Researcher with the Pattern and Image Analysis group at the Telecommunications Institute, which is a private non-profit research institution. His research interests include inverse problems, signal and image processing, pattern recognition, optimization, and remote sensing. He was and is involved in several national and international research projects and networks.

Dr. Bioucas-Dias is an Associate Editor of the IEEE TRANSACTIONS ON ImAge Processing, and he was an Associate Editor of the IEEE TRANSACTIONS ON CiRCUITS AND Systems. He is a Guest Editor of two IEEE special issues (IEEE TGRS and IEEE JSTARS). He was the General Co-Chair of the 3rd IEEE Workshop on Hyperspectral Image and Signal Processing: Evolution in Remote Sensing-Whispers'2011 and has been a member of program/technical committees of several international conferences. He has authored and co-authored many journal and conference papers.

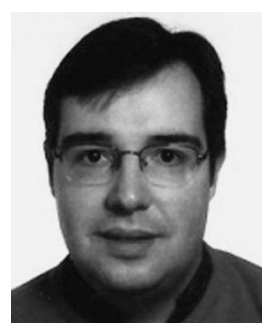

Antonio Plaza (M'05-SM'07) received the M.S. and $\mathrm{Ph} . \mathrm{D}$. degrees in computer engineering from the University of Extremadura, Caceres, Spain.

$\mathrm{He}$ was a Visiting Researcher with the Remote Sensing Signal and Image Processing Laboratory, University of Maryland Baltimore County, Baltimore, with the Applied Information Sciences Branch, Goddard Space Flight Center, Greenbelt, MD, and with the AVIRIS Data Facility, Jet Propulsion Laboratory, Pasadena, CA. He is currently an Associate Professor with the Department of Technology of Computers and Communications, University of Extremadura, where he is the Head of the Hyperspectral Computing Laboratory. He was the Coordinator of the Hyperspectral Imaging Network, a European project designed to build an interdisciplinary research community focused on hyperspectral imaging activities. He has been a Proposal Reviewer with the European Commission, the European Space Agency, and the Spanish Government. He is the author or coauthor of around 350 publications on remotely sensed hyperspectral imaging, including more than 70 journal citation report papers, 20 book chapters, and over 200 conference proceeding papers. His research interests include remotely sensed hyperspectral imaging, pattern recognition, signal and image processing, and efficient implementation of large-scale scientific problems on parallel and distributed computer architectures.

Dr. Plaza has coedited a book on high-performance computing in remote sensing and guest edited seven special issues on remotely sensed hyperspectral imaging for different journals, including the IEEE TRANSACTIONS ON GEOSCIENCE AND REMOTE SENSING (for which he serves as Associate Editor on hyperspectral image analysis and signal processing since 2007), the IEEE Journal of Selected Topics in Applied Earth Observations and Remote Sensing, the International Journal of High-Performance Computing Applications, and the Journal of Real-Time Image Processing. He has served as a reviewer for more than 280 manuscripts submitted to more than 50 different journals, including more than 140 manuscripts reviewed for the IEEE TRANSACTIONS on Geoscience And Remote Sensing. He has served as a Chair for the IEEE Workshop on Hyperspectral Image and Signal Processing: Evolution in Remote Sensing in 2011. He has also been serving as a Chair for the SPIE Conference on Satellite Data Compression, Communications, and Processing since 2009, and for the SPIE Remote Sensing Europe Conference on HighPerformance Computing in Remote Sensing since 2011. He is a recipient of the recognition of Best Reviewers of the IEEE Geoscience and Remote Sensing Letters in 2009 and a recipient of the recognition of Best Reviewers of the IEEE TRANSACTIONS ON GeOSCIENCE AND REMOTE SENSING in 2010. He is currently serving as Director of education activities for the IEEE Geoscience and Remote Sensing Society. 